NBSIR 85-3222

\title{
Polyadic Third-Order Lagrangian Tensor Structure and Second-Order Sensitivity Analysis With Factorable Functions
}

Richard H. F. Jackson

U.S. DEPARTMENT OF COMMERCE

National Bureau of Standards

Center for Applied Mathematics

Gaithersburg, MD 20899

and

Garth P. McCormick

Department of Operations Research

School of Engineering and Applied Science

George Washington University

Washington, DC 20052

August 1985

\section{Sponsored by}

U.S. DEPARTMENT OF COMMERCE

National Bureau of Standards

QC

100

.456

85-3222

1985 

U.S. DEPARTMENT OF COMMERCE

National Bureau of Standards

Center for Applied Mathematics

Gaiţhersburg, MD 20899

and

Garth P. McCormick

Department of Operations Research

School of Engineering and Applied Science

George Washington University

Washington, DC 20052

August 1985

Sponsored by

U.S. DEPARTMENT OF COMMERCE

National Bureau of Standards

Center for Applied Mathematics

Operations Research Division

Gaithersburg, MD 20899

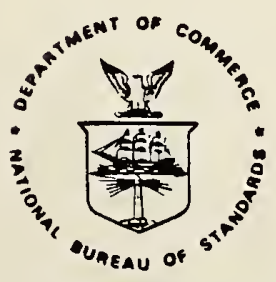

U.S. DEPARTMENT OF COMMERCE, Malcolm Baldrige, Secretary NATIONAL BUREAU OF STANDARDS, Ernest Ambler, Director 


\begin{abstract}
Second-order sensitivity analysis methods are developed for analyzing the behavior of a local solution to a constrained nonlinear optinization problem when the problem functions are perturbed slightly. Specifically, formulas Involving third-order tensors are given to compute second derlvatives of components of the local solution with respect to the problem parameters. When In addition, the problem functions are factorable, it is shown that the resulting tensors are polyadic in nature.
\end{abstract}

Reywords: Second-order sensitivity analysis, high-order methods, $\mathrm{N}^{\text {th }}$ derivatives, polyads, tensors. 
List of Tables........................................ v

List of Figures...................................... $v 1$

1. Introduction....................................... 1

2. The Speclal Structure of Tensors of Factorable Functions......... 8

2.1 Background and Notation............................. 8

2.2 The First and Second order Cases....................... 10

2.3 The $\mathrm{N}^{\mathrm{th}}$-Order Case................................. 14

3. Second-Order Sensitivity Analysis in Nonlinear Programing....... 19

3.1 Basic First-Order Sensitivity Results.................... 19

3.2 Development of the Second-Order Equation................. 23

3.3 Structure of the Three-Dimensional Arrays................ 28

3.4 Polyadics in Second-Order Sensitivity Analysis............. 35

3.4.1 The Dyadics in the Second-Order Terms.............. 35

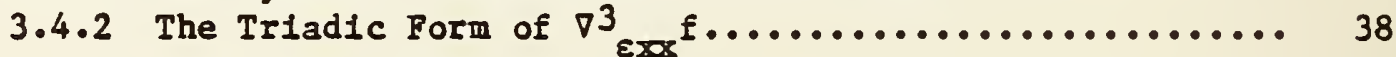

3.4 .3 The Triadic Form of $\nabla_{\mathrm{x} \varepsilon x^{f}}^{3} \ldots \ldots \ldots \ldots \ldots \ldots \ldots \ldots \ldots \ldots \ldots \ldots . \ldots 4$

3.4 .4 The Trladic From of $\nabla_{x \varepsilon x^{f}}^{3} \ldots \ldots \ldots \ldots \ldots \ldots \ldots \ldots \ldots \ldots \ldots . \ldots . \ldots 4$

3.5 Array Multiplication with Generalized Outer Product

Matrices..................................... 45

3.6 Parameter Tensors of Optimal Value Function.............. 49

3.7 Second-Order Sensitivity Results in Use................. 50

Bibliography........................................ 58 


\section{List of Tables}

Page

Table 1 Gradients of Factorable Function Forms................. 12

Table 2 Hessians of Factorable Function Forms................. 12

Table 3 Monadic and Dyadic Terms in Gradient and Hessian

of Illustrative Function......................... 13

Table 4 Dyadic and Triadic Terms in Hessian and Third Order

Tensor of Illustrative Function.................... 17

Table 5 Gradients of Factorable Function Forms in Sensitivity

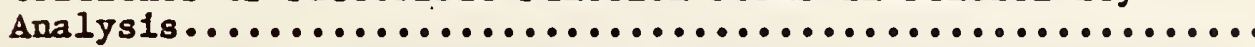

Table 6 Hessians of Factorable Function Forms in Sensitivity

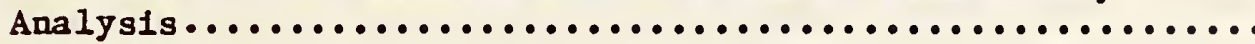

Table 7 Hessians with Respect to $x$ and $\varepsilon$ of Factorable Function

Forms in Sensitivity Analysis...................... 38

Table 8 Third-Order Tensors with Respect to $x, x$, and $\varepsilon$ of

Factorable Function Forms in Sensitivity Analysis......... 40

Table 9 Third-Order Tensors with Respect to $x, \varepsilon$, and $x$ of

Factorable Function Forms in Sensitivity Analysis.......... 42

Table 10 Third-Order Tensors with Respect to $x, \varepsilon$, and $\varepsilon$ of

Factorable Function Forms in Sensitivity Analysis......... 44 
Figure 1 Possible Orientations of a Matrix in Three-Space......... 26

Figure 2 Basic Partitioned Structure of $\nabla_{\varepsilon}$ N................... 29

Figure 3 Exploded View of Structural Detalls of $\nabla_{\varepsilon}$ N.............. 29

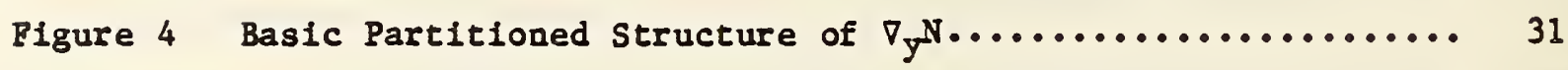

Figure 5 Exploded $\nabla$ iew of Structural Details of $\nabla_{y} N \ldots \ldots \ldots \ldots \ldots \ldots \ldots$

Figure 6 Basic Partitioned Structure of $\nabla_{\varepsilon}$ M................... 33

Figure 7 Exploded View of Structural Detalls of $\nabla_{\varepsilon}$ M............ 33

Figure 8 Basic Partitioned Structure of $\nabla_{y}$ M..................... 34

Figure 9 Exploded $\nabla$ iew of Structural Details of $\nabla_{y} M \ldots \ldots \ldots \ldots \ldots \ldots . . . . .34$

Figure 10 Three Ways to Multiply a Three-Dimensional Array by a Matrix..................................... 46 


\section{INT RODUCTION}

In an earlier paper (Jackson and McCormick (1984)) the structure taken by $\mathrm{N}$-dimensional arrays of $\mathrm{N}^{\text {th }}$ partial derivatives of the special class of factorable functions was examined. The $N$-dimensional arrays (or tensors as they are sometimes called) turn out to be computable naturally as the sum of generalized outer product matrices (polyads). For the benefit of the reader unfamiliar with polyads and factorable functions, some of the material in that paper is repeated here.

This natural polyadfc structure has important computational fmplications for solving problems associated with nonlinear programing. It means for example that with some minor modification to existing software routines, highorder derivatives can be calculated efficiently, making previously intractable techniques that require them, again worthy of consideration. In the dissertation by Jackson (1983) from which most of the material in this paper is taken, these implications were pursued for second-order sensitivity analysis and high-order methods for solving the problem:

$$
\begin{aligned}
& \operatorname{minimize} f(x), \\
& x \in R^{n} \\
& \text { subject to } g(x)>0,
\end{aligned}
$$

for $1=1, \ldots, m$, when $f(x)$ and $g(x)$ are factorable functions.

The ability to compute third derfvatives efficiently provides ready access to second-order nonlinear programming sensitivity information. In Section 3 of this paper, second-order sensitivity analysts methods are developed for analyzing the behavior of a local solution to (1.1) when the problem functions are perturbed slightly. Section 3 begins by summarizing results from first-order sensitivity analysis which provide formulas for the first derivatives of the components of the local solution with respect to the problem parameters. Also developed are formulas, imvolving third-order 
tensors, for computing the second derivatives of the local solution with respect to the problem parameters. In addition, the polyadic structure of the tensors is investigated and displayed, and techniques for manipulating these three-dimensional arrays, capitalizing on this special structure, are developed. In general, this type of array mantpulation is straightforward but time-consuming and requires significant computer storage. It is shown that these difficulties are ameliorated when the spectal structure of factorable functions is exploited. Examples of the use of these formulas for estimating the solution to perturbed problems using Taylor series approximations are also given.

Loosely, a factorable function is a multivariable function that can be written as the last of a fintte sequence of functions, in which the first $n$ functions in the sequence are just the coordinate variables, and each function beyond the $n^{\text {th }}$ is a sum, a product, or a single-variable transformation of previous functions in the sequence. More rigorously, let $\left[f_{1}(x), f_{2}(x), \ldots\right.$, $f_{L}(x)$ ] be a finite sequence of functions such that $f_{1}: R^{n} \rightarrow R$, where each $f_{i}(x)$ is defined according to one of the following rules.

Rule 1. For $i=1, \ldots, n, f_{i}(x)$ is the value of the $i$ th Euclidean coordinate:

$$
f_{1}(x)=x_{1}
$$

Rule 2. For $i=n+1, \ldots, L, f_{1}(x)$ is formed using one of the following compos-

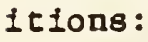

$$
\begin{aligned}
& \text { a.) } f_{1}(x)=f_{j}(1)(x)+f_{k}(1)(x) \text {; or } \\
& \text { b.) } f_{1}(x)=f_{j}(1)(x) \cdot f_{k(1)}(x) \text {; or } \\
& \text { c.) } f_{1}(x)=T_{1}\left[f_{j}(1)(x)\right] \text {; }
\end{aligned}
$$

where $j(i)<1, k(1)<1$, and $T_{1}$ is a function of a single variable. Then $f(x)=f_{L}(x)$ is a factorable function and $\left[f_{1}(x), f_{2}(x), \ldots, f_{L}(x)\right]$ is a 
factored sequence. Thus a function, $f(x)$, will be called factorable if $1 t$ can be formed according to Rules 1 and 2 , and the resulting sequence of functions will be called a factored sequence or, at times, the function written in factored form.

Although it is not always immediately grasped, the concept of a factorable function is actually a very natural one. In fact, it is just a formallzation of the natural procedure one follows in evaluating a complicated function. Consider for example the function

$$
f(x)=a^{T} x[\operatorname{sinb} x]\left[\operatorname{expc} c^{T} x\right]
$$

where $a, b, c$ and $x$ are $(2 \times 1)$ vectors. The natural approach to evaluat1ng this function for specific values of $x_{1}$ and $x_{2}$ is first to compute the quantities within the parentheses, then to apply the sine and exponential functions, and finally to multiply the three resulting quantities. This might be done in stages as follows.

$$
\begin{array}{ll}
f_{1}=x_{1} & f_{8}=f_{6}+f_{7} \\
f_{2}=x_{2} & f_{9}=c_{1} f_{1} \\
f_{3}=a_{1} f_{1} & f_{10}=c_{2} f_{2} \\
f_{4}=a_{2} f_{2} & f_{11}=f_{9}+f_{10} \\
f_{5}=f_{3}+f_{4} & f_{12}=\sin \left(f_{8}\right) \\
f_{6}=b_{1} f_{1} & f_{13}=\exp \left(f_{11}\right) \\
f_{7}=b_{2} f_{2} \quad f_{14}=f_{5} \cdot f_{12}
\end{array}
$$

This is one possible factored sequence for the function in (1.3). 
For completeness, we also note here that a Factorable Programming problem may be compactly (though less transparently) written as

$$
\begin{aligned}
& \underset{\text { minfmize }}{x \in R^{n}} f_{L}(x) \\
& \text { subject to } b_{\perp}<f_{I}(x)<u_{I},
\end{aligned}
$$

for $1=1, \ldots, L-1$, where it is possible that $\ell_{I}=-\infty$ and/or $u I=+\infty$, and where $f_{\perp}(x)=x_{1}$, for $1 \leqslant n$, and $f_{I}(x)$ is defined recursively for $I>n$ as

$$
f_{\perp}(x)=\sum_{p=1}^{\perp-1} U_{p}^{ \pm}\left[f_{p}(x)\right]+\sum_{p=1}^{1-1} \sum_{q=1}^{p} v_{q, p}^{\perp}\left[f_{p}(x)\right] w_{p, q}^{\perp}\left[f_{q}(x)\right]
$$

where $U, V$, and $W$ are functions of a single varfable.

In order to apprectate fully the value of factorable functions the concept of an outer product matrix must be introduced. An ( $m \times n$ ) matrix $A$ is called an outer product matrix if there exists a scalar $\alpha$, an (m $x$ 1) vector $a$, and an ( $n \times 1)$ vector $b$ such that

$$
A=a \alpha b^{T} \text {. }
$$

The expression $a a b^{T}$ is called an outer product or a dyad. Note that a dyad

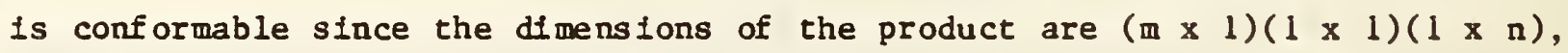
which yields the (m $x \mathrm{n}$ ) outer product matrix $A$ as desired. A useful property of outer product matrices is that, if kept as dyads, matrix multiplication with them is simplified to inner products alone, saving the computations required to form the matrices involved. For example,

$$
\begin{aligned}
& A c=a \alpha\left[b^{T} c\right], \\
& d^{T} A=\left[d^{T} a\right] \alpha b^{T}, \text { and } \\
& A F=a \alpha\left[b^{T} F\right],
\end{aligned}
$$

where $c$ is $(n \times 1), d$ is $(m \times 1)$ and $F$ is $(n \times m)$. 
It is well-known (see for instance McCormick (1983)) that factorable functions possess two very spectal properties that can be explofted to produce efficlent (fast and accurate) algorithms: 1 ) once written in factorable form, the1r gradients and Hessians may be computed exactly, automatically, and efficiently; and 11) their.Hessians occur naturally as sums of dyads whose vector factors are gradients of terms in the factored sequence. The first of these properties has eased the task of providing the derivatives of a nonlfnear programming problem to a computer code that solves it, and has the potential eventually to trivialize it. The second has obviated the task of multiplying a matrix by a vector, reducing it to a series of inner products, as noted above, which in many cases results in less effort.

Since the discovery of factorable functions, the theory of Factorable Programming has been further developed and refined in a variety of ways. Ghaemi and McCormick (1970) developed a computer code (FACSUMT), which processes the functions in a factorable program and provides the interface to the SUMT nonlinear programming code developed originally by Mylander et al. (1971). An early version of this code is described in Pugh (1972). Recently the routines from FACSUMT that process the problem functions in factorable form have been separated out into a "stand-alone" package (FACTIN) that can be used with any nonlinear programing system to provide automatically the values of the functions, gradients and Hessians at a point. The basic requirement of FACTIN is that the user write the functions of the problem in factorable form.

Once the problem is written in factorable form, the routines in FACT IN automatically calculate the exact first and second derivatives of the functions for use in the optimization algorithm. 
It is fmportant to understand that the dertvative calculations performed by the FACTIN code are not estimations, but mathematically exact calculations. Furthermore, they are also compact, since factored sequences mimic hand calculations, and thus this technique is different from symbolic manipulation techafques for differentiation, which tend to produce large amounts of code. The techniques used in Factorable Programming are effictent exploitations of the spectal structure inherent in factorable functions and their partial derivative arrays. Moreover, while it is true that some symbolic differentiaters also can recognize functions which can be described stmilarly as a sequence of rules, each of which can be differentiated, the similarity ends there. Such symbolic differentiaters continue to differentiate the rules, without exploitIng the polyadic structure of the result. See,e.g., Kedem (1980), Rall (1980), Wengert (1964), Refter and Gray (1967), Hillstrom (1982), and Warner (1975). It is this latter effort which provides the real value of factorable functions, and which therefore separates the two techniques. More on Factorable Programming codes and the Factorable Programming system (FACTPROG) under development at the National Bureau of Standards is given in Jackson and MeCormick (1984).

Further extensfons of Factorable Programming theory were provided by Shayan (1978), who developed an automatic method for computing the m $^{\text {th-order }}$ directional dertvative of a factorable function and noted that the efficiency of a solution technique can be evaluated when the functions are factorable by counting basic operations and bastc functions. This is a more accurate measure of effictency than the technique of counting the number of "equivalent function evaluations" discussed by Miele and Gonzalez (1978). 
As was mentioned earlier, Hessians of factorable functions possess a natural dyadic structure which can be exploited. This structure was used in Emami (1978) to develop a matrix factorization scheme for obtaining a general1zed Inverse of the Hessian of a factorable function. Ghotb (1980) also capitalized on this structure and provided formulae for computing a generalized Inverse of a reduced Hessian when it is given in dyadic form. Sofer (1983) has extended this last concept further, by utilizing the dyadic structure to obtain computationally efficient techniques for constructing a generalized Inverse of a reduced Hessian and updating it from iteration to iteration. Another direction was pursued by DeSilva and McCormick (1978), who developed the formulae and methodology to utilize the input to general nonlinear programs in factorable form to perform first-order sensftivity analysis on the solution vector.

Some last comments on notation are required. There are unavoidable complications in the theory that follows that require subscripted subscripts. In some cases these are used. In other cases, subscripted subscripts are replaced with subscript functions. For example, $i_{j} \rightarrow i(j)$. The choice in each case was made on the basis of clarity of resulting formulae. Also in what follows, all vectors are assumed to be colum vectors, and, where not otherwise stated, differentiation is with respect to the vector $x=\left(x_{1}, x_{2}\right.$, $\left.\ldots, x_{n}\right)^{T}$. Lastly, we use $\partial$ and $\nabla$ to indicate partial dffierentiation, and $d$ and $D$ indicate total differentiation. 


\section{THE SPECIAL STRUCTURE OF TENSORS OF FACTORABLE FUNCT IONS}

\subsection{Background and Notation}

One fundamental value of factorable functions lies in the simple and computationally efficient forms that result for their Hessians. In tact factorable programming is based on the existence of, and the simplified operations that result from, these simple forms. The seminal result, (F1acco and McCormick (1968), pp 184-188), is that the Hess 1an of a factorable function can be written as the sum of dyads, or outer products, of gradients of functions in the factored sequence. As was shown in Jackson and McCormick (1984) and w1ll be summar1zed here, this bas1c result may be generalized, but First it is necesssary to generalize the concepts of Hessian and dyad.

Let $A \in R^{\left(n_{1} \times \cdots \times n_{N}\right)}$, and let $A_{1_{1}}, \ldots, 1_{N}$ denote the $\left(1_{1}, \ldots, i_{N}\right)$ th element of this array. For the purposes of this paper, A is called the Nth-order tensor of a multivariable function $f(x)$ if

$$
A_{1}, \ldots, 1_{N}=\partial^{N} f(x) / \partial x_{1_{N}} \ldots \partial x_{1}
$$

Note that gradfents and Hessians are tensors of order 1 and 2 respectively. An N-dimensional array A is called a generalized outer product matrix if there exists a scalar $\alpha$, and an ordered set of vectors $a_{1}, \ldots$, an (where each $a_{k}$ is $\left(n_{k} \times 1\right)$ ) such that each element of $A$ is generated by the product of the scalar $\alpha$ and certain spectfic elements of the vectors $a, \ldots$, an as follows

$$
A_{1_{1}}, \ldots, 1_{N}=\alpha * a_{1,1_{1}} * \ldots * a_{N}, \pm_{N},
$$

for $1_{1}=1, \ldots, n_{1} ; \ldots ; i_{N}=1, \ldots, n_{N}$, where $a_{k}, 1_{k}$ represents the $\left(i_{k}\right)$ th element of the $\left(n_{k} \times 1\right)$ vector ak. 
The scalar and set of vectors which generate a generalized outer product matrix taken together are called a polyad and are written

$$
\left(\alpha: a_{1} \cdots a_{N}\right)
$$

where order is important, 1.e. the vector in position $j$ is associated with the $j^{\text {th }}$ dimension. A polyad containing $\mathbf{N}$ vector factors is called an $\mathrm{N}$-ad. Also, an expression containing a sum of polyads is called a polyadic, and an expression containing a sum of $\mathrm{N}$-ads is called an $\mathrm{N}$-ad1C. (The actual add1tion here is performed as a direct sum of the associated generalized outer product matrices.) When vector factors in a polyad are repeated, exponential notation is used, as, e.g., In the case of the symmetric N-ad, $\left(\alpha:[a]^{N}\right)$. Note that the representation of a generalized outer product matrix by a polyad is not unfque. For example, $\left(\alpha / \gamma:\left[a_{1} \gamma\right] \cdots a_{N}\right)$ generates the same $N$-dimensıonal array of numbers as does (2.1) for any nonzero scalar $\gamma$. Finally, note that a 2-ad of the form ( $\alpha: a b)$ is equivalent to the more familiar dyad of the form $a a b^{T}$, and the two w111 be used interchangeably.

Because the concepts of generalized outer product matrix and polyad are so fundamental to what follows, an example is included to help in understanding them. Consider first the dyad $\left(10: a_{1} a_{2}\right)$ where $a_{1}=(4,3,2,1)^{T}$, and $a_{2}=(3,2,1)^{T}$. The general1zed outer product matr $1 x$ of order 2 generated by this dyad is the $(4 \times 3)$ array

$$
\left[\begin{array}{ccc}
120 & 80 & 40 \\
90 & 0 & 60 \\
60 & 40 & 30 \\
30 & 20 & 10
\end{array}\right]
$$

If the vector $a_{3}=(2,1)^{\mathrm{T}}$ is added to form the $\operatorname{tr} 1 \mathrm{ad}\left(10: \mathrm{a}_{1} \mathrm{a}_{2} \mathrm{a}_{3}\right)$, the result 
is a generalized outer product matrix of order 3 with dimensions $(4 \times 3 \times 2)$. To form this three-dimensional array, the outer product between the matrix above and the vector $a_{3}$ is formed. Thus the matrix above is multiplied by 2 to obtain the front matrix in the three-dimensional array and by 1 to obtain the back matrix. These are:

$\left[\begin{array}{rrr}240 & 160 & 80 \\ 180 & 120 & 60 \\ 120 & 80 & 40 \\ 60 & 40 & 20\end{array}\right]$

\subsection{The First and Second Order Cases}

In this section, the special polyadic structure of the gradient and the Hessian of a factorable function is exhibited.

\section{Theorem 1. (Monadic Gradients)}

Let $f(x)$ be a factorable function in $R n$, let $\left[f_{1}(x), f_{2}(x), \ldots, f_{L}(x)\right]$ be a factored sequence for $f(x)$, and assume that all functions are once continuously differentiable. Then the gradient $\nabla f(x)=\nabla f_{L}(x)$ can be written as a sum of outer product matrices of the form $\left(\alpha: a_{1}\right)$, where $a_{1}$ is a gradient of a factored-sequence function and the scalar a is composed of a product of factored-sequence functions and first derivatives of the single-varfable transformations, $T_{i}$, used in the factored sequence.

Proof. See Jackson (1983).

The result given in Theorem 2 below is a formalization of a result which appeared without proof in McCormick (1983). 


\section{Theorem 2. (Dyadic Hessians)}

Let $f(x)$ be a factorable function in $R^{n}$, let $\left[f_{1}(x), f_{2}(x), \ldots, f_{L}(x)\right.$ ] be a factored sequence for $f(x)$, and assume that all functions are twice continuously differentiable. Then the Hessian $\nabla 2 f(x)=\nabla 2 f_{L}(x)$ can be written as a sum of outer product matrices of the form $\left(\alpha: a_{1} a_{2}\right)$, where $a_{1}$ and $a_{2}$ are gradients of factored-sequence functions and the scalar $\alpha$ is composed of a product of factored-sequence functions and first and second derivatives of the single-variable transformations, $T_{i}$, used in the factored sequence. Proof. See Jackson (1983).

Although the proofs of Theorems 1 and 2 are not Included, the monadic and dyadic structure of the gradient and Hessian of a factorable function are exhibited by displaying the gradients and Hessians of the forms in (1.2) as in Tables 1 and 2 .

In order to clarify these concepts, consider again the illustrative function in (1.3). Table 3 is a display of the gradient and Hessian of this function. The entries in each colum are the summands in the expressfons for the gradient and Hessian. For example,

or, in polyadic notation

$$
\begin{gathered}
\nabla f=\left[\sin \left[b^{T_{x}}\right]\right]\left[\exp \left[c^{T_{X}}\right]\right] a+\left[a_{x}\right]\left[\cos \left[b^{T}\right]\right]\left[\exp \left[c T_{x}\right]\right] b \\
+\left[a^{T} x\right]\left[\sin \left[b^{T} x\right]\left[\exp \left[c^{T} x\right]\right] c\right.
\end{gathered}
$$

$$
\begin{gathered}
\nabla f=\left(\left[\sin \left[b^{T_{X}}\right]\right]\left[\exp \left[c^{T} x\right]\right]: a\right)+\left(\left[a_{X}\right]\left[\cos \left[b^{T} x\right]\right]\left[\exp \left[c T_{x}\right]\right]: b\right) \\
+\left(\left[a^{T_{X}}\right]\left[\sin \left[b^{T} x\right]\left[\exp \left[c^{T} x\right]\right]: c\right) .\right.
\end{gathered}
$$

The table also illustrates the left-to-right, tree-like structure of the der:vatives involved. From the table it can be seen that both the gradient and Hessian naturally have the polyadic structure discussed above. Notfce too that the vectors in the monads and dyads are drawn from the set $\{a, b, c\}$, each of which is the gradient of a factored sequence function in (1.4). 
TABLE 1

GRADIENTS OF FACTORABLE FUNCTION FORMS*

\begin{tabular}{|c|c|c|}
\hline Rule & $f_{1}$ & $\nabla f_{1}$ \\
\hline 1 & $x_{i}$ & $e_{1}$ \\
\hline $2 a$ & $f_{j(1)}+f_{k(1)}$ & $\nabla f_{j(1)}+\nabla f_{k(1)}$ \\
\hline $2 b$ & $f_{j(1)} \cdot f_{k(1)}$ & $\nabla f_{k}(1) f_{j(1)}+\nabla f_{j(1)} f_{k(1)}$ \\
\hline $2 c$ & $T_{1}\left[f_{j(1)}\right]$ & $\nabla f_{j(1)} \dot{T}_{1}\left[f_{j(1)}\right]$ \\
\hline
\end{tabular}

TABLE 2

HESSIANS OF FACTORABLE FUNCTION FORMS*

\begin{tabular}{|c|c|c|}
\hline Rule & $f_{1}$ & $\nabla^{2} f_{1}$ \\
\hline 1 & $x_{1}$ & $0_{n \times n}$ \\
\hline $2 a$ & $f_{j(1)}+f_{k(1)}$ & $\nabla^{2} f_{j(1)}+\nabla^{2} f_{k(1)}$ \\
\hline $2 b$ & $f_{j(1)} \cdot f_{k(1)}$ & $f_{j(1)} \nabla^{2} f_{k(1)}+\nabla f_{k(1)} f_{j(1)}^{T}$ \\
\hline $2 c$ & $T_{1}\left[f_{j(1)}\right]$ & $\dot{T}_{1}\left[f_{j(1)}\right] \nabla^{2} f_{j(1)}+\nabla f_{j(1)} \tilde{T}_{i}\left[f_{j(1)}\right] \nabla f_{j(1)}+\nabla f_{j(1)} \nabla f_{k(1)}$ \\
\hline
\end{tabular}

*Notation: $\dot{T}[f]=\partial T / \partial f, \dot{T}[f]=\partial 2 T / \partial f^{2}$, and $e_{1}=$ the 1 th unit vector $\pm n R n$. 
TABLE 3

MONADIC AND DYADIC TERMS IN GRADIENT

AND HESSIAN OF ILLUST RATIVE FUNCTION*

\begin{tabular}{|c|c|c|}
\hline f & $\begin{array}{c}\nabla f \\
\text { (summands) }\end{array}$ & $\begin{array}{c}\nabla^{2} \mathrm{f} \\
\text { (summands) }\end{array}$ \\
\hline \multirow{3}{*}{$a^{T} x \sin \left[b^{T} x\right] \exp \left[c^{T} x\right]$} & $\left(\sin \left[b^{T} x\right] \exp \left[c^{T} x\right]: a\right)$ & $\begin{array}{l}\left(\cos \left[b^{T} x\right] \exp \left[c^{T} x\right]: a b\right) \\
\left(\sin \left[b^{T} x\right] \exp \left[c^{T} x\right]: a c\right)\end{array}$ \\
\hline & $\left(a^{T} x \cos \left[b T_{x}\right] \exp \left[c^{T} x\right]: b\right)$ & $\begin{array}{r}\left(\cos \left[b^{T} x\right] \exp \left[c^{T} x\right]: b a\right) \\
\left(-a^{T} x \ln \left[b^{T} x\right] \exp \left[c^{T} x\right]: b b\right) \\
\left(a^{T} x \cos \left[b^{T} x\right] \exp \left[c^{T} x\right]: b c\right)\end{array}$ \\
\hline & $\left(a^{T} x \sin \left[b^{T} x\right] \exp \left[c^{T} x\right]: c\right)$ & $\begin{array}{r}\left(\sin \left[b^{T} x\right] \exp \left[c^{T} x\right]: c a\right) \\
\left(a^{T} x \cos \left[b^{T} x\right] \exp \left[c^{T} x\right]: c b\right) \\
\left(a^{T} x \operatorname{1n}\left[b^{T} x\right] \exp \left[c^{T} x\right]: c c\right)\end{array}$ \\
\hline
\end{tabular}

${ }^{*}$ N.B. Since the meaning is clear, we have dropped a level of parentheses in these expressions to be able to fit the table on one page. The same comment holds for Table 4 . 


\subsection{The Nth-Order Case}

The next theorem is fundamental to operations with higher derivatives of factorable functions, and provides the necessary tool to use in proving that all tensors of factorable functions are polyadics in gradients of factoredsequence functions.

Theorem 3. (Differentiation of Polyads)

Let $f(x)$ be a factorable function in $R^{n}$, let $\left[f_{I}(x), f_{2}(x), \ldots, f_{L}(x)\right]$ be 1ts factored sequence, and assume that all functions are members of $c^{N+1}$. Consider the $\mathrm{N}$-ad

$$
\left(a: a_{1} a_{2} \ldots a_{N}\right) \text {, }
$$

where the $a_{j}$ are gradients of factored-sequence functions and $\alpha$ is a scalar composed in general of a product of factored-sequence functions and derivatives (no higher than order $N$ ) of the single-variable transformations used in forming the factored sequence. The gradient of this $\mathrm{N}$-ad is the sum of $(\mathrm{N}+\mathrm{I})-$ ads, each term of which has the structure defined above.

Proof. Because the proof of this theorem 1llustrates the technique of polyadic differentiation, its salient features are included here. For convenience, write $f_{1}$ for $f_{1}(x)$. Then the scalar $\alpha$ is of the form

$$
a=\pi n_{\ell},
$$

where

$$
n_{\ell}=\mathrm{f}_{\mathrm{r}(\ell)} \text { or } \partial \mathrm{k}(\ell)\left\{\mathrm { T } _ { \mathrm { s } ( \ell ) } \left[\mathrm{f}_{\mathrm{r}(\ell)]\} / \partial \mathrm{f}_{\mathrm{r}(\ell)} \mathrm{k}(\ell)}\right.\right. \text {, }
$$

for values of $r(\ell), s(l)$, and $k(\ell)$ which respectively define, for the $\ell^{\text {th }}$ factor of $\alpha$, the factored-sequence function, the transformation, and the 
level of the derivative of the transformation used. Note that $k(l)<N$. By the chain rule of differentiation,

$$
\nabla \alpha=\sum_{\ell} \gamma_{\ell<\ell}
$$

where

$$
\gamma_{\ell}=\frac{\alpha}{n_{l}}, \text { or }-\frac{\alpha}{n_{l}} \cdot \partial^{k(l)+1}\left\{\operatorname{Tr}_{S}(l)\left[f_{r}(l)\right]\right\} / \partial \mathrm{f}_{r(l)}^{k(l)+1} \text {, }
$$

and

$$
c_{\ell}=\nabla f_{r}(\ell) \cdot
$$

Furthermore, since the $a_{j}$ in the $\mathrm{N}$-ad are gradients of factored-sequence functions, $\nabla a_{j}$ can be written by the previous theorem as the sum of outer product matrices of order 2 in gradients of factored-sequence functions as follows :

$$
\nabla a_{j}=\sum_{(p, q) \varepsilon I_{j}} b_{p}^{\beta} p q^{b_{q}}=\sum_{(p, q) \varepsilon I_{j}}\left(B_{p q}: b_{p} b_{q}\right),
$$

where $I_{j}=\left\{(p, q) \mid b_{p} \beta_{p q} b_{q}^{T}\right.$ is a term in the dyadic representation of $\left.\nabla a_{j}\right\}$ and $B_{p q}=B_{q p}$, is of the same form as $\alpha$. Then, by straightforward term-byterm differention and collection of terms (see Jackson and McCormick (1984)), It can be shown that the gradient of the $\mathrm{N}$-ad is

$$
\begin{aligned}
\nabla\left(\alpha: a_{1} a_{2} \cdots a_{N}\right)= & \sum_{\ell}\left(\gamma_{\ell: a_{1}} \cdots a_{N} c_{\ell}\right) \\
& +\sum_{(p, q) \varepsilon I_{1}}\left(\alpha B_{p q}: b_{p} \cdots a_{N} b_{q}\right) \\
& \cdot \\
& \cdot \\
& +\sum_{(p, q) \varepsilon I_{N}}\left(\alpha B_{p q}: a_{1} \cdots a_{N-1} b_{p} b_{q}\right),
\end{aligned}
$$

which is a sum of $(\mathrm{N}+1)$-ads in gradients of factored sequence functions with leading scalars of the proper form, and the result is proven. 
Now the main theorem on the structure of high-order tensors of factorable functions can be given.

\section{Theorem 4. (Polyadic Tensors)}

Let $f(x)$ be a factorable function in $R^{n}$, let $\left[f_{1}(x), f_{2}(x), \ldots, f_{L}(x)\right]$ be a factored sequence for $f(x)$, and assume that $f(x) \varepsilon C^{N}$ and $f_{1}(x) \varepsilon C^{N}$, for $1=1, \ldots$, L. Then the $N^{t h}$-order tensor of $f(x)$ can be written as the sum of generalized outer product matrices of the following special form. Let $\left(\alpha: a_{1} \cdots a_{N}\right)$ be a polyad associated with one of the outer product matrices. Then each ak is the gradient of some function in the factored sequence, and the scalar $\alpha$ is a product of functions in the factored sequence and derivatives of the single-variable transformations used in defining the functions in the sequence. Only derivatives $\partial k_{T}[f] / \partial f k$, for $1<k<N$, are used.

Proof. The proof is a strafghtforward application of Theorem 3 and induction; the reader is referred to Jackson and McCormick (1984) for the detalls.

At this point another example is offered to help illustrate these ideas. To keep matters as simple as possible, the same fllustrative function in (1.3), whose gradient and Hessian were displayed in Table 3, will be used. Calculation of the third-order tensor of this function is a therapeutic exercise, but only the final result is given here in Table 4. The terms in the Hessian from Table 3 are also included in $\mathrm{T}$ able 4 to make it easter to see the left-to-right, tree-like connections among successive gradient terms. Notice that the vectors that make up each triad are once again drawn from the set $\{a, b, c\}$ and that the scalar multiples in the triads are products of factored-sequence functions (e.g., $a^{T} x, \sin \left[b T_{x}\right], \exp \left[c^{T}\right]$ ) and first and second derivatives of the transformations $\left(e_{\cdot} \cdot g \cdot, \cos \left[b \mathrm{~T}_{\mathrm{X}}\right],-\sin \left[\mathrm{b} \mathrm{T}_{\mathrm{X}}\right]\right)$. Not $\pm c e$ 
TABLE 4

DYADIC AND TRIADIC TERMS IN HESSIAN AND THIRD ORDER TENSOR OF ILLUST RAT IVE FUNCT ION

\begin{tabular}{|c|c|}
\hline $\begin{array}{c}\nabla^{2} \mathbf{f} \\
\text { (summands) }\end{array}$ & $\begin{array}{c}\nabla 3_{\mathrm{f}} \\
\text { (summands) }\end{array}$ \\
\hline$\left(\cos \left[b^{T} x\right] \exp \left[c^{T} x\right]: a b\right)$ & $\begin{array}{r}\left(-\sin \left[b^{T} x\right] \exp \left[c^{T} x\right]: a b b\right) \\
\left(\cos \left[b^{T} x\right] \exp \left[c^{T} x\right]: a b c\right)\end{array}$ \\
\hline$\left(s \pm n\left[b^{T} x\right] \exp \left[c^{T} x\right]: a c\right)$ & $\begin{array}{l}\left(\cos \left[b^{T} x\right] \exp \left[c^{T} x\right]: a c b\right) \\
\left(\sin \left[b^{T} x\right] \exp \left[c^{T} x\right]: a c c\right)\end{array}$ \\
\hline$\left(\cos \left[b^{T} x\right] \exp \left[c^{T} x\right]: b a\right)$ & $\begin{array}{r}\left(-\sin \left[b^{T} x\right] \exp \left[c^{T} x\right]: b a b\right) \\
\left(\cos \left[b^{T} x\right] \exp \left[c^{T} x\right]: b a c\right)\end{array}$ \\
\hline$\left(-a^{T} x s \operatorname{nn}\left[b T_{x}\right] \exp \left[c^{T} x\right]: b b\right)$ & $\begin{array}{r}\left(-\sin \left[b^{T} x\right] \exp \left[c^{T} x\right]: b b a\right) \\
\left(-a^{T} x \cos [b T x] \exp \left[c^{T} x\right]: b b b\right) \\
\left(-a^{T} x \sin \left[b^{T} x\right] \exp \left[c^{T} x\right]: b b c\right)\end{array}$ \\
\hline$\left(a^{T} x \cos \left[b^{T} x\right] \exp \left[c^{T} x\right]: b c\right)$ & 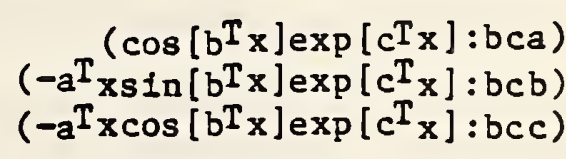 \\
\hline$\left(\sin \left[b^{T} x\right] \exp \left[c^{T} x\right]: c a\right)$ & $\begin{array}{l}\left(\cos \left[b^{T} x\right] \exp \left[c^{T} x\right]: c a b\right) \\
\left(\sin \left[b^{T} x\right] \exp \left[c^{T} x\right]: c a c\right)\end{array}$ \\
\hline$\left(a^{T} x \cos \left[b^{T} x\right] \exp \left[c^{T} x\right]: c b\right)$ & $\begin{array}{r}\left(\cos \left[b^{T} x\right] \exp \left[c^{T} x\right]: c b a\right) \\
\left(-a^{T} x \sin \left[b^{T} x\right] \exp \left[c^{T} x\right]: c b b\right) \\
\left(-a^{T} x \cos \left[b^{T} x\right] \exp \left[c^{T} x\right]: c b c\right)\end{array}$ \\
\hline$\left({ }^{T} T_{x s i n}\left[b^{T} x\right] \exp \left[c^{T} x\right]: c c\right)$ & $\begin{array}{r}\left(\sin \left[b^{T} x\right] \exp \left[c^{T} x\right]: c c a\right) \\
\left(-a^{T} x \cos \left[b^{T} x\right] \exp \left[c^{T} x\right]: c c b\right) \\
\left(a^{T} x \cos \left[b^{T} x\right] \exp \left[c^{T} x\right]: c c c\right)\end{array}$ \\
\hline
\end{tabular}


too that the only new calculations needed to form $\nabla^{3} f$ are the third derivatives of the single variable transformations: sin, cos, and exp.

There are several computational advantages to be stressed here. One advantage is a result of the symmetry of partial derivatives, i.e., $\partial 3 f / \partial x_{i} \partial x_{j} \partial x_{k}=\partial 3 f / \partial x_{j} \partial x_{k} \partial x_{i}$, etc. Because of this symmetry, every triad in the $\nabla^{3}$ f colum in $T$ able 4 whose vector factors are the same, disregarding order, has the same assoctated scalar. For example, abb, bba, and bab, all have the same associated scalar: $\left[-\sin \left[b^{T} x\right]\right]\left[\exp \left[c^{T} x\right]\right]$. Thus only six distinct scalars need to be computed to form $\nabla^{3} \mathrm{f}$. Moreover, because of what may be called the "persistence property" of the derivatives of sin and exp in this example, the calculation of these six scalars is also simplified. That is, all derivatives of exp are equal and alternating derivatives of sin are equivalent except for their signs. This too can be exploited to reduce computational effort. Finally, since the vector factors in the triads are members of the same set from which the dyads in the Hessian and the monads in the gradient are formed, a computer code that calculates these need only store $a, b$, and $c$, and a set of pointers for each monad, dyad, and triad Indicating which vector is required for each position. Of course, these pointers can be packed to save storage.

The current versions of the factorable programming input routines (FACSUMT and FACTIN) take advantage of each of the above in computing the gradients and Hessians. Work is underway to extend these codes to capitalize on the polyadic structure of factorable function tensors and compute highorder derivatives in the efficient manner discussed above. See Jackson and McCormlck (1984) for more on this current work. 
3. SECOND-ORDER SENSITIVITY ANALYSIS IN NONLINEAR PROGRAMMING

\subsection{Basic First-order Sensitivity Results}

One application of the results in Section 2 is in obtaining high-order sensitfvity information for nonlinear programming problems, although only the second-order case is considered in this paper. Sensitivity analysis in nonlinear programming is concerned with analyzing the behavior of a local solution when the problem functions are perturbed slightly. This perturbation might be due to an inexactness with which certain parameter values in the problem are calculated or because the optimization model was parameterized and one is interested in the solution for a variety of values of the parameters. For additional information on this topic, see Armacost and Fiacco (1974), Armacost and Fiacco (1978), Fiacco (1980) and especially Fiacco (1983) and its excellent biblfography. The parametric problem is written

$$
\begin{aligned}
& P(\varepsilon): \quad \begin{array}{l}
\operatorname{minfmize} \\
x \varepsilon R^{n}
\end{array} \quad f(x, \varepsilon), \\
& \text { subject to } g_{1}(x, \varepsilon)>0,
\end{aligned}
$$

for $1=1, \ldots, m$, where $\varepsilon$ is an $(r \times .1)$ vector of parameters. The more general version of the sensitivity problem includes equality constraints, but these are not included here for simplicity. The ideas and results presented in this section generalize readily to the equality-constrained problem.

The essence of sensitivity analysis in nonlinear programming is the application of the Implicit Function Theorem (see, e.g. Bliss (1946) to the Karush-Kuhn-Tucker (KKT) necessary conditions for the problem, $P(\varepsilon)$, $\pm n(3.1)$. 
First, define the Lagrangian for $P(\varepsilon)$ as

$$
L(x, u, \varepsilon)=f(x, \varepsilon)-\sum_{i=1}^{m} u_{1} g_{1}(x, \varepsilon) \text {. }
$$

Then, assuming continuous differentiability in $x$ of the problem functions, the KKT conditions for $P(\varepsilon)$ are that there exists a feasible point, $x$, for (3.1) and associated vector of Lagrange multipliers, $u$, such that

$$
\begin{aligned}
\nabla L(x, u, \varepsilon) & =0, \\
u_{1} g_{1}(x, \varepsilon) & =0, \\
u_{1} & >0,
\end{aligned}
$$

for $1=1, \ldots, \mathrm{m}$.

The statement of the first-order sensitivity results given in Theorem 6 below also requires that the second-order sufficient conditions (SOSC) hold at a particular solution $\hat{x}$, of $P(\hat{\varepsilon})$ (which is just $(3.1$ ) for a speciffed vector of parameter values, $\hat{\varepsilon})$. These conditions may be written as follows.

\section{Theorem 5. (SOSC)}

Let $\hat{\mathbf{x}}$ be a feastble point for $P(\hat{\varepsilon})$ and assume that the functions of $P(\hat{\varepsilon})$ are twice-continuously differentiable in $x$ in a neighborhood of $\hat{x}$. Let $(\hat{x}, \hat{u}, \hat{\varepsilon})$ be a triple that satisfies the KKT conditions in (3.2) and define

and

$$
B=\left\{1 \mid g_{1}(\hat{x}, \hat{\varepsilon})=0\right\} \text {, }
$$

$$
D=\left\{1 \mid \hat{u}_{1}>0\right\} \text {. }
$$

Further, suppose that

$$
\mathrm{d}^{\mathrm{T}} \nabla^{2} \mathrm{~L}(\hat{\mathrm{x}}, \hat{\mathbf{u}}, \hat{\varepsilon}) \mathrm{d}>0 \text {, }
$$

for all $d \neq 0$, such that

and

$$
\mathrm{d}^{\mathrm{T}} \nabla \mathrm{g}_{1}(\hat{x}, \hat{\varepsilon})>0, \text { for all } \perp \varepsilon \mathrm{B},
$$

$$
\mathrm{d}^{\mathrm{T}} \nabla g_{1}(\hat{x}, \hat{\varepsilon})=0, \text { for all } 1 \varepsilon \mathrm{D} \text {. }
$$

Then $\hat{x}$ is a strict local minimizer for $P(\hat{\varepsilon})$. 
The earliest known reference to these conditions is Pennisi (1953), although it was almost 15 years (see McCormick (1967), and Fiacco and McCormick (1968)) before they were mre fully developed and exploited.

The following theorem can be viewed as the basic result in nonlinear programing senstivity analysis.

Theorem 6. (First-order Sensitivity)

Let $\hat{x}$ be a feasible point for $P(\hat{\varepsilon})$ and assume that

1.) the functions in (3.1) are twice-continuously differentiable in $x$ and the cross-partial derivatives exist and are jointly continuous in $x$ and $\varepsilon$ in a netghborhood of $(\hat{x}, \hat{\varepsilon})$;

11.) the second-order sufficient conditions (Theorem 5) for a local minfmum of $P(\hat{\varepsilon})$ hold at $\hat{x}$, with associated Lagrange multipliers $\hat{u}$,

111.) the gradients of the binding constraints, $1 . e ., \nabla g_{1}(\hat{x}, \hat{\varepsilon}), 1 \varepsilon$, are Inearly independent; and

iv.) $\hat{u}_{1}>0$ for all $1 \in$ B.

Then, for $\varepsilon$ in asufficiently small neighborhood of $\hat{\varepsilon}$, there exists a unique, once-continuously differentiable vector function

$$
y(\varepsilon)=\left[x(\varepsilon)^{T}, u(\varepsilon)^{T}\right]^{T},
$$

satisfying the KKT conditions for $P(\varepsilon)$, with

$$
y(\hat{\varepsilon})=\left[x(\hat{\varepsilon})^{\mathrm{T}}, \mathrm{u}(\hat{\varepsilon})^{\mathrm{T}}\right]^{\mathrm{T}},
$$

such that $x(\varepsilon)$ is a locally unique isolated minimizer for $P(\varepsilon)$. Furthermore, the first partial derivatives of $x(\varepsilon)$ and $u(\varepsilon)$ with respect to $\varepsilon$ can be obtained from the equation

$$
Y=-M^{-1} N \text {, }
$$


where

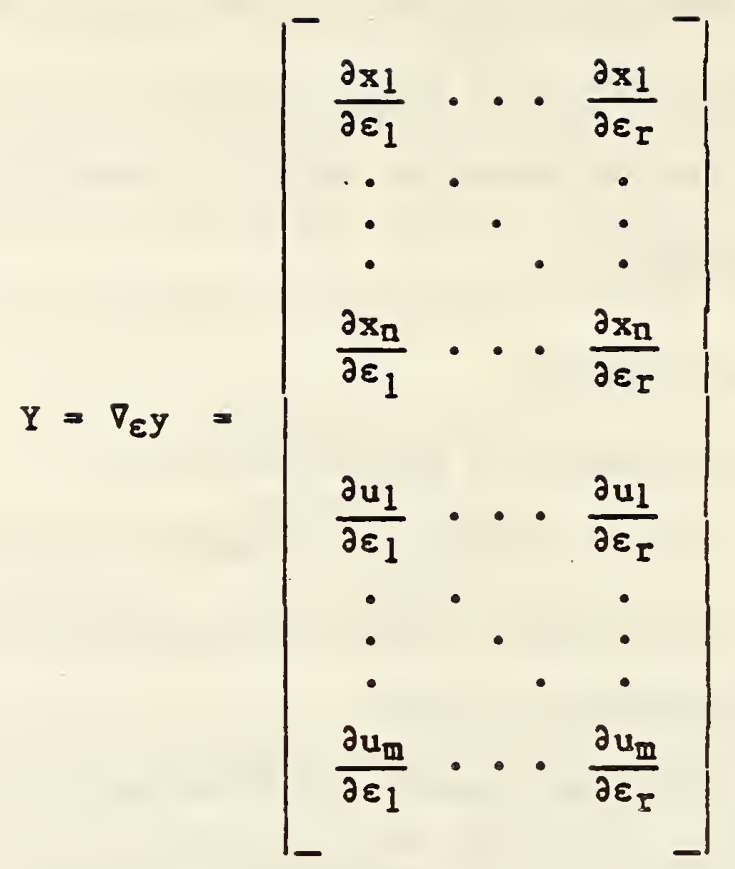

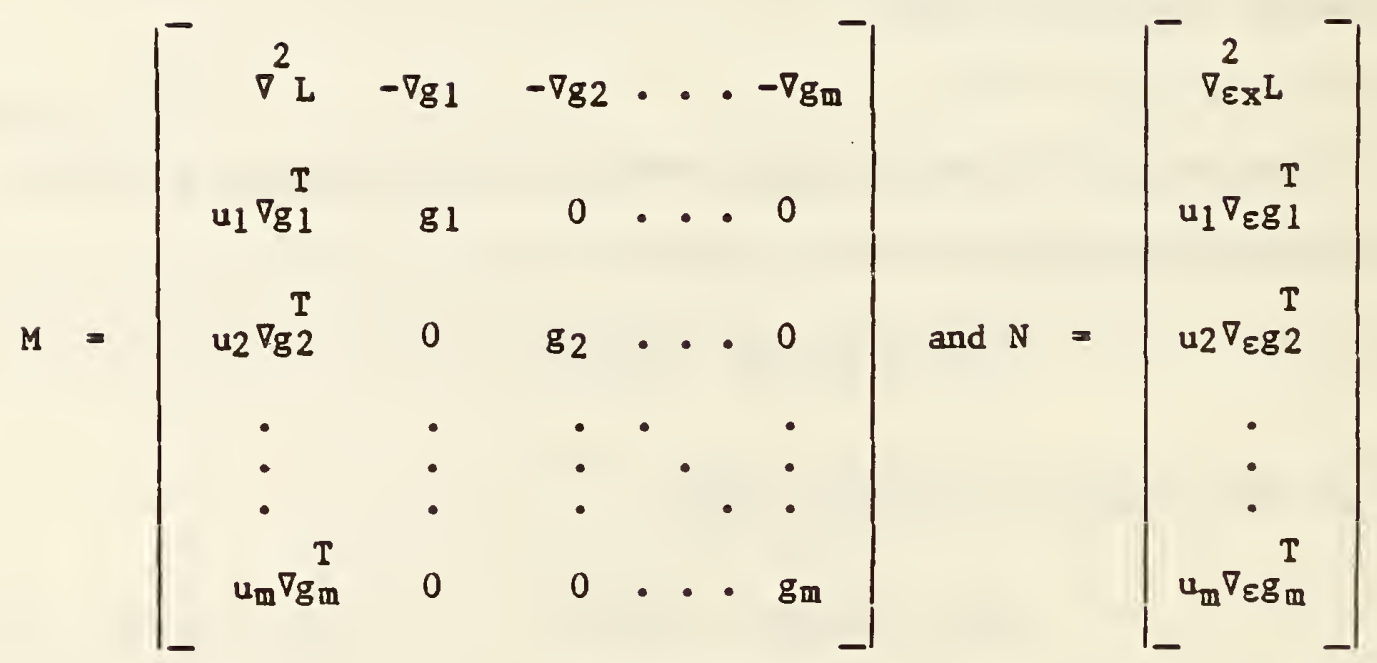

with all quantities in $\mathrm{M}$ and $\mathrm{N}$ evaluated at $\left(\hat{\mathrm{x}}^{\mathrm{T}}, \hat{\mathrm{u}}^{\mathrm{T}}\right)^{\mathrm{T}}$. Proof. See Fłacco (1983). 


\subsection{Development of the Second-Order Equation}

The result in (3.3) provides a direct way of calculating first-order sensitivity information and is the point of departure for the work given here, which begins with the following theorem due to Flacco (1983).

Theorem 7. (Higher-Order Sens $\pm t \pm v \pm t y$ )

Let $\hat{x}$ be a feastble point for $P(\hat{\varepsilon})$ and assume conditfons ( 1 ) through

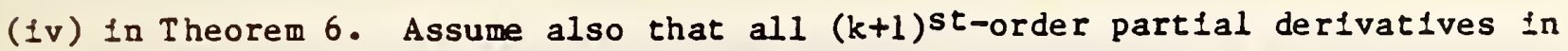
$x$ and all $(k+1) s t$-order cross partfal derfvatives in $x$ and $\varepsilon$ exist and are jointly continuous in $x$ and $\varepsilon$ in a neighborhood of $(\hat{x}, \hat{\varepsilon})$. Then in a sufflclently small netghborhood of $\hat{\varepsilon}$, the vector function $y(\varepsilon)$ is $k$ times continuously differentiable.

Proof. The proof follows directly from the fact that if the Jacobtan, M, of the KKT conditions in (3.2) Is nonsingular, and if the functions involved possess the appropriate degree of differentiablity, the Implicit Function Theorem (see Blfss (1946), p. 270) guarantees the existence of the higherorder partial derivatives. Nonsingularfty of $M$ for (3.2) was shown in Fiacco and McCormick (1968). Thus the theorem is proved by direct applfcation of the Implicte Function Theorem.

If this high-order sensitivity information is to be used, it is necessary also to develop a convenfent mechanism for calculating it. This is done next for the case when $k=2$. Consider the matrix equation in (3.3) and rewrtte it as

$$
M Y=-N
$$

The $(1, j)^{\text {th }}$ element of this matricial equation is

$$
\sum_{s} M_{1 s} Y_{s j}=-N_{1 j},
$$


and the total derivative of this equation is obtained using the product rule as follows:

$$
\sum_{s}\left\{M_{i s} \frac{\partial Y_{s} j}{\partial \varepsilon_{k}}+Y_{s j} \frac{d M_{i s}}{d \varepsilon_{k}}\right\}=-\frac{d N_{i} j}{d \varepsilon_{k}},
$$

or

$$
-\sum_{s} M_{i s} \frac{\partial Y_{s} j}{\partial \varepsilon_{k}}=\sum_{s} \frac{d M_{i s}}{d \varepsilon_{k}} Y_{s j}+\frac{d N_{i} j}{d \varepsilon_{k}}
$$

Next, the chain rule is required in finding the right-hand-side (rhs) in (3.4) since each element of the $M$ and $N$ matrices is a function of each element of $x$, $u$, and $\varepsilon$. Hence,

$$
\frac{d M_{i} \underline{s}}{d \varepsilon_{k}}=\sum_{t} \frac{\partial M_{i s}}{\partial y_{t}} \frac{\partial y_{t}}{\partial \varepsilon_{k}}+\frac{\partial M_{i s}}{\partial \varepsilon_{k}}
$$

and

$$
\frac{d N_{1 j}}{d \varepsilon_{k}}=\sum_{\varepsilon} \frac{\partial N_{1 j}}{\partial y_{t}} \frac{\partial y_{t}}{\partial \varepsilon_{k}}+\frac{\partial N_{1 j}}{\partial \varepsilon_{k}}
$$

Using these and the fact that $Y_{s j}=\partial y_{s} / \partial \varepsilon_{j}$, the rhs in (3.4) can thus be written

$$
\sum_{s}\left[\left[\frac{\partial M_{i s}}{\partial y_{t}} Y_{t k}+\frac{\partial M_{i}}{\partial \varepsilon_{k}}\right] Y_{s j}+\sum \frac{\partial N_{i j}}{\partial y_{t}} Y_{t k}+\frac{\partial N_{i j} j}{\partial \varepsilon_{k}}\right.
$$

Now consider the left-hand-side (1hs) in (3.4) more closely. It can be written

$$
-\sum_{s} M_{i s} \frac{\partial^{2}}{\partial \varepsilon_{k}}-\frac{y_{s}}{\partial \varepsilon_{j}},
$$

which ts of the form

$$
-\sum_{s} M_{i s} A_{s j k}
$$

which in turn gives the $(i, j, k) t h$ element of the three-dimensional array that results from the matrix multiplication of $M$ and the $k$ th (counting from front

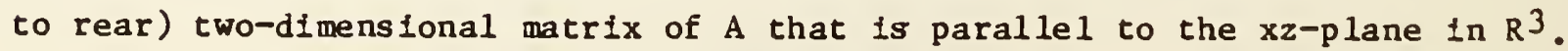


Observe that $\partial^{2} y_{1} / \partial \varepsilon_{k} \partial \varepsilon_{j}$ can be obtained by premultiplying both sides of (3.4) by $\mathrm{H}=\mathrm{M}^{-1}$. Then

$$
\frac{\partial^{2} y_{1}}{\partial \varepsilon_{j}}-\frac{1}{\partial \varepsilon_{k}}=-\sum H_{i r}\left\{\sum_{s}\left[\sum \frac{\partial M_{r s}}{\partial y_{t}} \frac{\partial y_{t}}{\partial \varepsilon_{k}}+\frac{\partial M_{r s}}{\partial \varepsilon_{k}}\right] \frac{\partial y_{s}}{\partial \varepsilon_{j}}+\sum_{t} \frac{\partial N_{r} j}{\partial y_{t}} \frac{\partial y_{t}}{\partial \varepsilon_{k}}+\frac{\partial N_{r} j}{\partial \varepsilon_{k}}\right],
$$

which is the $(1, j, k)$ th element of the three-dimenstonal array of second partial derfvatives of $y=\left[x(\varepsilon)^{T}, u(\varepsilon)^{T}\right]^{T}$ with respect to $\varepsilon$.

It is desired next to write (3.5) using array notation. Because these operations are performed in three-space, however, some new notation must be developed before this is rewritten. In order to motivate the new notation, consider a multivariable function $f(x)$. It is perhaps clear in this case what is meant by $\nabla f, \nabla 2 \mathrm{f}$ and $\nabla 3 \mathrm{f}$; 1.e., $\nabla \mathrm{f}$ is a vector that is written down the page, $\nabla^{2}$ requires taking the gradient of each element of $\nabla f$ and writing that result across the page to form a two-dimensional matrix, and thus to form $\nabla{ }^{3} \mathrm{f}$ one would take the gradient of each element of $\nabla 2 f$ and write that result into the third dimension ( 1 nto the page, say). Hence if $M$ is a matrix, it should be clear what is meant by $\nabla M$; $1 . e .$, take the gradient of each element of $M$ and write the result into the third dimension.

But, if $y$ is a vector $1 n R^{2}$, there are three possible orfentations parallel to the coordinate planes in $R^{3}$ for the matrix usually notated as $\nabla y$. These are shown* in Figure 1. In order clearly to differentiate among these, the notation " $\nabla\{\cdot\}$ " w1ll be used. Thus $\nabla\{\cdot\}$ operates on the argument by taking its gradient into the third dimension. Note that

$$
\nabla y \neq \nabla\{y\} \neq \nabla\left\{y^{T}\right\}
$$

Whereas the elements of these vectors are the same, their orfentations in

*A11 graphs were produced using DATAPLOT as described in Filliben (1981). 


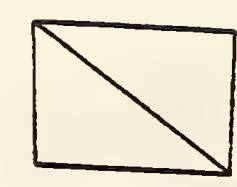

Q

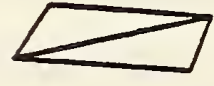

$\nabla \mathbf{y}$

$\nabla\{y\}$

$\nabla\left\{y^{\top}\right\}$

\section{FIGURE 1}

\section{POSSIBLE ORIENTATIONS OF A MATRIX IN THREE-SPACE}

three-space are not. These, too, are shown in Figure 1. The same comments apply with regard to total differentiation, for which the notation $D\{\cdot\}$ will be used. This notation, of course, is only used for an operation from $R^{2}$ to $\mathrm{R}^{3}$; analogs exist for higher dimensions.

Now with this notation, the matrix form of (3.5) is written:

$$
\nabla_{\varepsilon \varepsilon y}^{2}=-M^{-1}\left[\nabla_{y} M \nabla_{\varepsilon}\{y\} \nabla_{\varepsilon y}+\nabla_{\varepsilon} M \nabla_{\varepsilon y}+\nabla_{y N} \nabla_{\varepsilon}\{y\}+\nabla_{\varepsilon} N\right] \text {, }
$$

where, letting $p=(n+m)$,

$$
\begin{array}{lll}
\nabla_{\varepsilon \varepsilon y}^{2} \text { is }(p \times 5 \times r) & \nabla_{\varepsilon y} \text { is }(p \times r \times 1) \\
M^{-1} \text { is }(p \times p \times 1) & \nabla_{\varepsilon} M \text { is }(p \times p \times r) \\
\nabla_{y} M \text { is }(p \times p \times p) & \nabla_{y} N \text { is }(p \times r \times p) \\
\nabla_{\varepsilon}(y\} \text { is }(p \times 1 \times r) & \nabla_{\varepsilon N} \text { is }(p \times r \times r)
\end{array}
$$

and the multiplication in three-space is carried out so that each array within

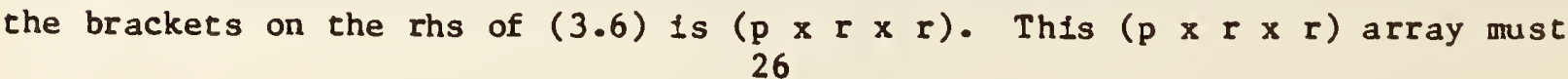


then be premultiplied by $M^{-1}$, a ( $\left.p \times p \times 1\right)$ array. This multiplication is effected by premultiplying each of the $r$ matrices of dimension ( $p r$ ) by $M^{-1}$, resulting $1 n r$ matrices of dimension ( $\left.p \times r\right)$, or a ( $\left.p \times r \times r\right)$ array agata, as required by $\nabla_{\varepsilon \varepsilon y}$. More on three-dimensional array multiplication (including graphical depictions) is given in Section 3.5 .

Observe that (3.3) could have been differentiated directly using the techntques for differentiation of matrices as given in Marlow (1978). He uses Kronecker products and a special two-dimensional representation of threedimenstonal arrays for performing this kind of differentiation. A disadvantage of this approach is that whatever spectal structure that exists in the three-dimensional arrays is lost in the process. This "structural integrity" is maintained in the approach given here, allowing the more detailed analysis given in the next section.

There has been some previous work in second-order sensitivity analysis. Dembo (1982) derived a computationally efficient technique for getting an approximation to the second derivative with respect to $\varepsilon$ of the vector function $y$, correct to terms of order two. By contrast, (3.6) is exact. Also, a result for the geometric programming problem for the case where $\varepsilon$ is a scalar was provided by Kyparisis (1983).

The material in the remainder of this section addresses the issue of computational efficiency when the problem functions are factorable. Sections 3.3 and 3.4 are admittedly rather detailed. The reason for including them is twofold: to fllustrate the spectal polyadic structure of the tensors in nonlinear programming sensitivity analysis, and to stimulate more research In these areas. It is not necessary to wade through this matertal for each 
second-order sensitivity calculation. Ultimately this will be performed automatically by the Factorable Programing system of programs befing developed at the National Bureau of Standards.

\subsection{Structure of the Three-Dimens ional Arrays}

While the formula in (3.6) may be mathematically succinct, it may not be obvious how the polyadic structure of derivatives of factorable functions can assist in its calculation. To understand how these calculations are performed, it is necessary to investigate further the structure of the threedimensional arrays involved. These are: $\nabla_{y} M, \nabla_{\varepsilon} M, \nabla_{y} N$, and $\nabla_{\varepsilon} N$. Since $\nabla_{\varepsilon^{N}}$ is the simplest of these, it is considered first. Strictly speaking, the $(i, j, k)^{\text {th }}$ element of $\nabla_{\varepsilon^{N}}$ is

$$
\left(\nabla_{\varepsilon} N\right)_{i j k}=\left\{\begin{array}{cl}
\frac{\partial^{3} L}{\partial \varepsilon_{k} \partial \varepsilon_{j} \partial x_{1}} & 1<n \\
u_{i-n} \frac{\partial^{2} g_{i-n}}{\partial \varepsilon_{k} \partial \varepsilon_{j}} & , \quad 1>n .
\end{array}\right.
$$

This can be thought of as taking the gradient with respect to $\varepsilon$ of each element of $\mathrm{N}$ into the third dimension, and thus can be pictured as a partitioned rectangular parallelepiped as shown in Figure 2. The partitioning, which is due to the parts in $y$, separates $\nabla_{E} N$ into an 3 "upper" part which is $\nabla_{\varepsilon \varepsilon \times L}$ and a "lower" part which is just a stack of constraint Hessians with respect to $\varepsilon$. This more detalled structure is shown in the exploded view of $\nabla_{\varepsilon^{N}}$ given In Figure 3. 


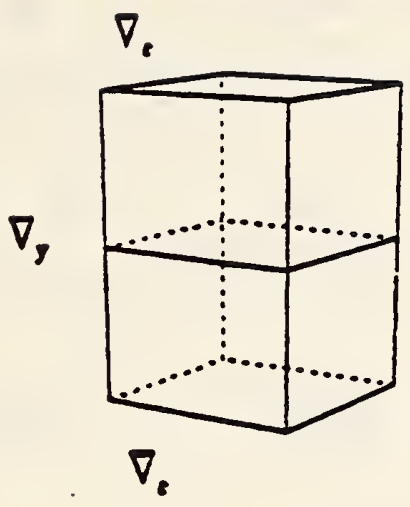

FIGURE 2

BASIC PARTITIONED STRUCTURE OF $\nabla N$

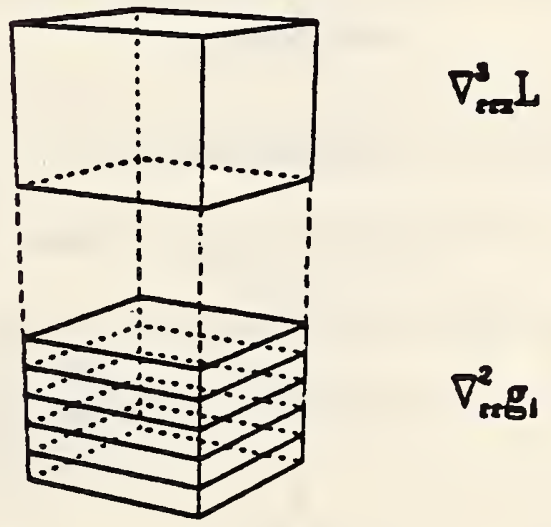

FIGURE 3

EXPLODED VIEW OF STRUCTURAL DETAILS

OF $\nabla N$ 
The next simplest array to portray is $\nabla_{y} N$. This is a three-dimensional array that has four parts, again a result of the two parts in $y$, arranged as shown in Figure 4. These partitions are described mathematically as:

$$
\left(\nabla_{y} N\right) i j k=\left\{\begin{array}{cc}
\frac{\partial^{3} L}{\partial x_{k} \partial \varepsilon_{j} \partial x_{i}}, & 1<n, k<n \\
u_{i-n} \frac{\partial^{2} g_{i-n}}{\partial x_{k} \partial \varepsilon_{j}}, & 1>n, k<n \\
-\frac{\partial^{2} g_{k-n}}{\partial \varepsilon_{j} \partial x_{i}} & , \quad 1<n, k>n \\
\frac{\partial g_{1-n}}{\partial x_{j}}, & i>n, k>n, i=k \\
0 \quad & \text { otherwise. }
\end{array}\right.
$$

These are depicted graphically in the exploded view in Figure 5, where the different orfentations of the various matrices and vectors in three-space are more easily grasped.

The next three-dimensional array to consider is $\nabla_{\varepsilon}{ }^{M}$. This too is partitioned into four smaller three-dimensional arrays, but with an orfentation that differs from $\nabla_{y} N$. The basic structure of the parts is shown in Figure 6 , and each element of the array is defined in the following equations:

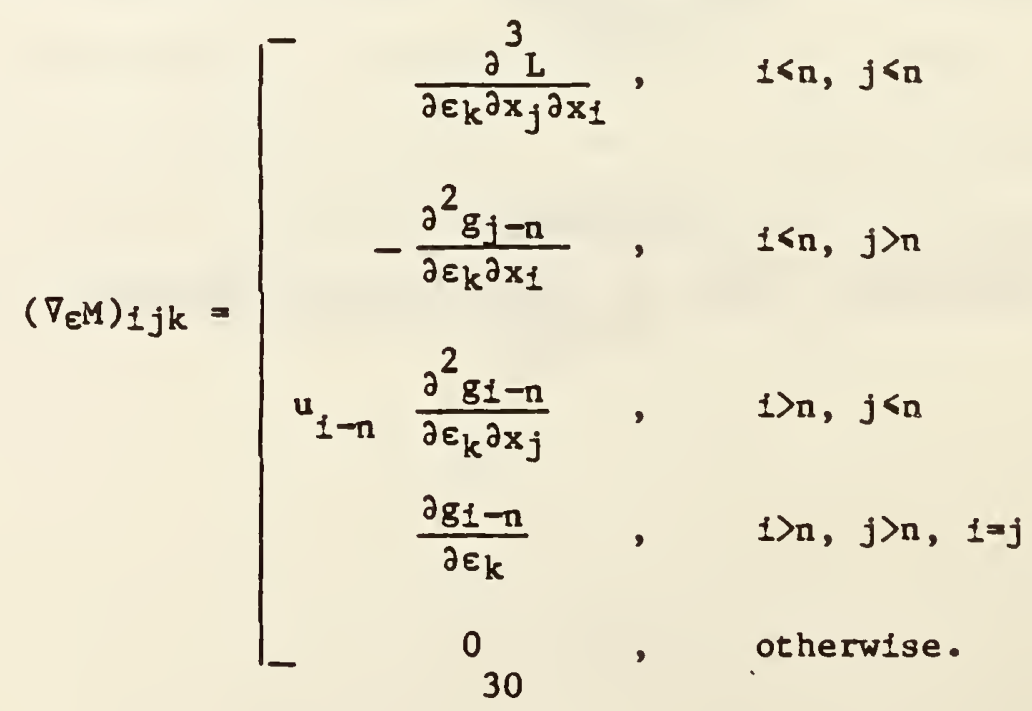




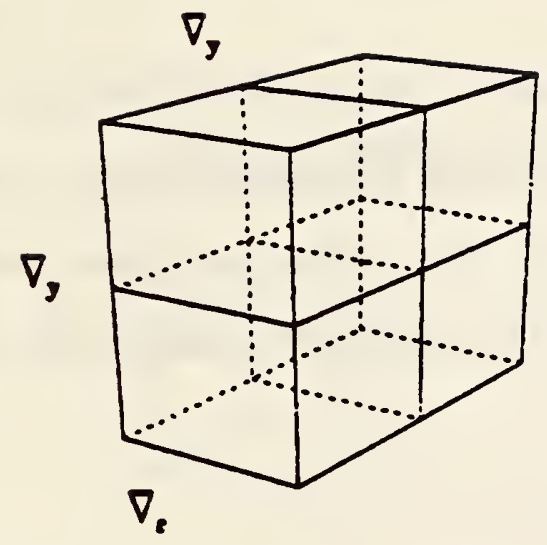

FIGURE 4

BASIC PARTITIONED STRUCTURE OF $\nabla_{3} \mathrm{~N}$

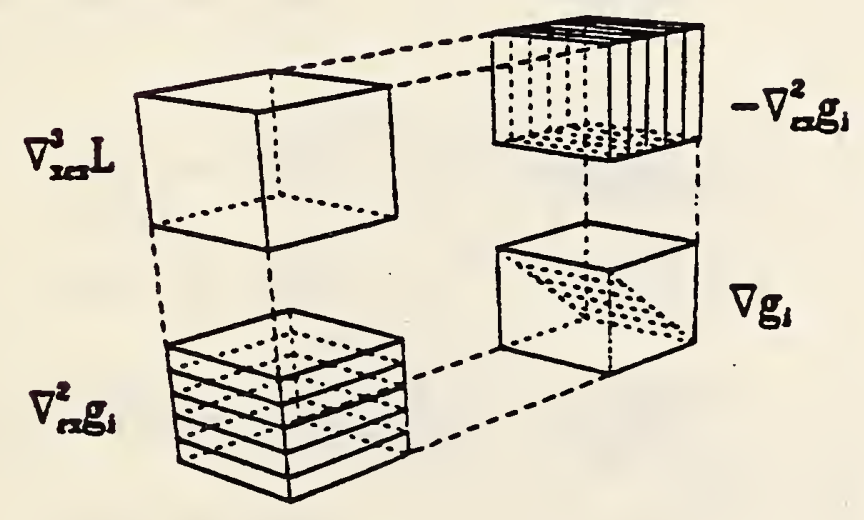

FIGURE 5

EXPLODED VIEW OF STRUCTURAL DETAILS OF $\nabla_{s} N$ 
The three-dimensional orientation of these matrices and vectors is shown in more detall in Figure 7.

The last three-dimensional array to be depicted is $\nabla_{Y M}$, the most complicated, with eight parts that result from differentiating three ifmes with respect to the partitioned vector $y$. The eight parts are shown in Figure 8 , and the mathematical statement of the $(i, j, k)$ th element for each case is given be low.

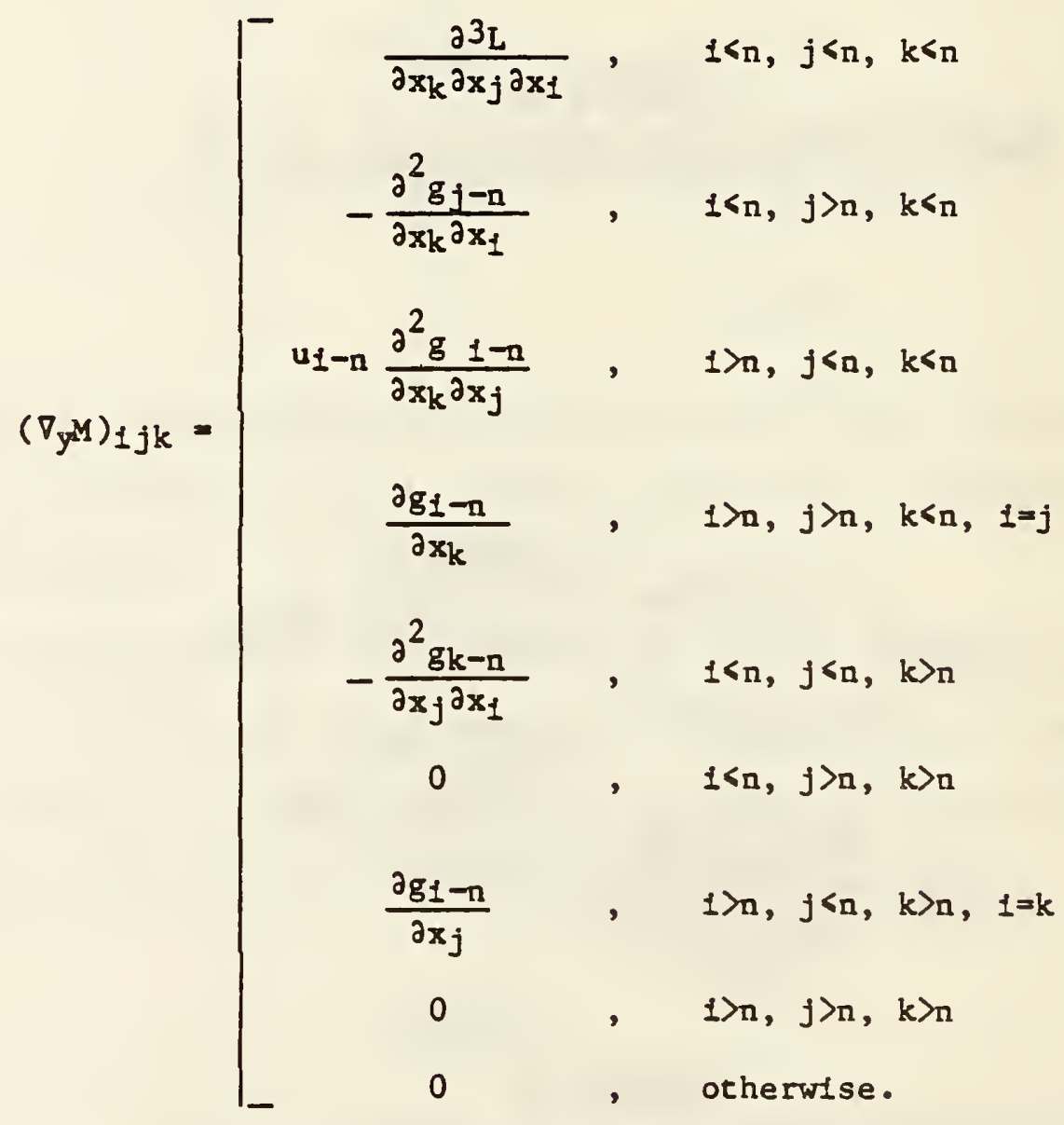

And finally, these structures are shown in detail in Figure 9. 


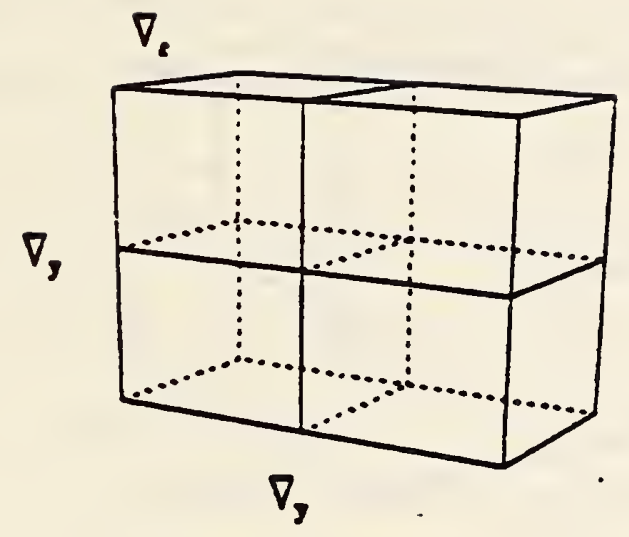

FIGURE 6

BASIC PARTITIONED STRUCTURE OF $\nabla_{\mathrm{M}} \mathrm{M}$

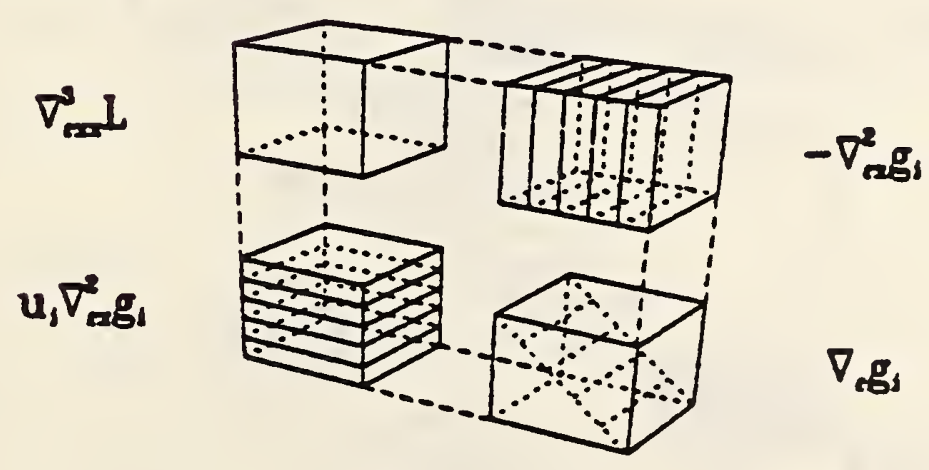

FIGURE 7

EXPLODED VIEW OF STRUCTURAL DETAILS OF $\nabla_{M} M$ 


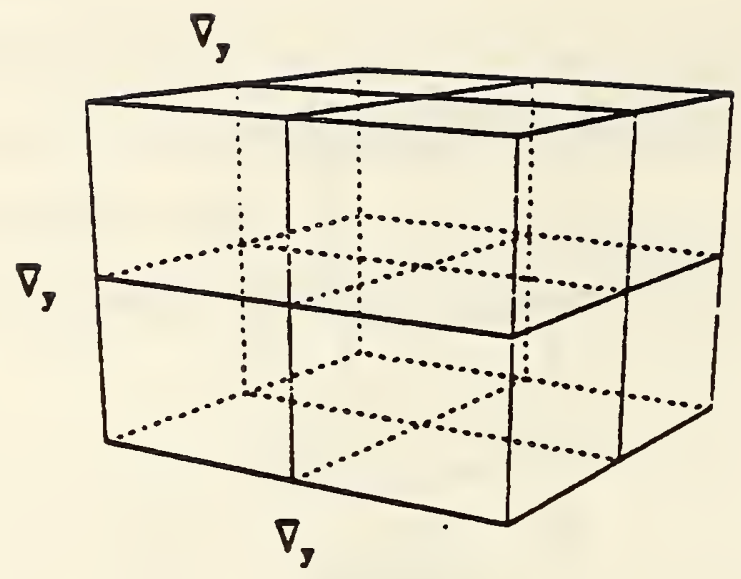

FIGURE 8

BASIC PARTITIONED STRUCTURE OF $\nabla_{\text {S }}$

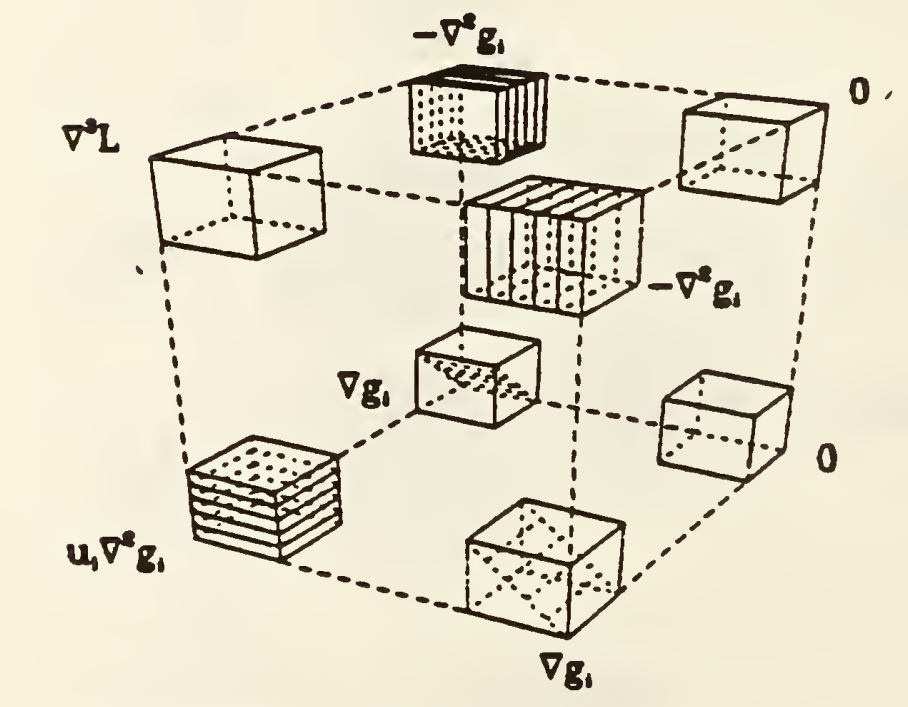

FIGURE 9

EXPLODED VIEW OF STRUCTURAL DETAILS OF $\nabla, M$ 


\subsection{Polyadics in Second-Order Sensitivity Analysis}

\subsubsection{The Dyadics in the Second-Order Terms}

Although the material in the previous section provided insight into the three-dimensional structure of the second-order sensttivity analysis formula In (3.6), it stıll may not be clear how the natural polyadic structure of tensors of factorable functions can assist in the evaluation of the formula. To see this, it is first necessary to show that each substructure exh 1 bited in Figures $3,5,7$, and 9 , is a polyadic, and then to demonstrate how the multiplication with $\nabla_{\varepsilon y}$ (or $\nabla_{\varepsilon}\{y\}$ ) is to be carried out. This section addresses the former of these activities by providing proofs that factorable functions of $(x, \varepsilon)$ have polyadic derivatives. Section 3.5 addresses the latter.

First notice that if the functions of the problem given in (3.1) are factorable in $x$ and $\varepsilon$, so too is the Lagrangian of that problem factorable in $x$ and $\varepsilon$. Then the task of this section reduces to considering some function $f(x, \varepsilon)=f_{L}(x, \varepsilon)$, with factored sequence $\left[f_{1}(x, \varepsilon), f_{2}(x, \varepsilon), \ldots, f_{L}(x, \varepsilon)\right]$ formed using the following modification to the rules given in $(1.2)$.

Rule 1. For $1<\mathrm{n}$,

$$
f_{1}(x, \varepsilon)=x_{1}
$$

Rule 2. For $1>n$, etther

$$
\begin{aligned}
& \text { a.) } f_{1}(x, \varepsilon)=f_{j}(1)(x, \varepsilon)+f_{k}(1)(x, \varepsilon) \text {; or } \\
& \text { b.) } f_{1}(x, \varepsilon)=f_{j}(1)(x, \varepsilon) \cdot f_{k(1)}(x, \varepsilon) \text {; or } \\
& \text { c.) } f_{1}(x, \varepsilon)=T_{1}\left[f_{j}(1)(x, \varepsilon), \varepsilon\right] \text {; }
\end{aligned}
$$

where $j(1)<1$, and $k(1)<1$, and the dertvatives wtth respect to $\varepsilon$ of the $T$, are themselves factorable functions in $\varepsilon$. For conventence, in the rest of this section, let $j(1)=j, k(1)=k$, and drop the arguments $(x, \varepsilon)$ and $\left[f_{j}(1)(x, \varepsilon)\right]$. Thus, e.g・, $T_{1}\left[f_{j}(1)(x, \varepsilon), \varepsilon\right]+T_{1}$. 
The gradients with respect to $x$ of the forms in (3.7) are given in Table 5. Calculation of the Hessians with respect to $x$ of these forms is straightforward and the result is given in Table 6. Notice that there is no difference between Table 6 and Table 2. Therefore Theorem 2 applies here and it is possible to write $\nabla^{2} \mathrm{f}(\mathrm{x}, \varepsilon)$ as a sum of dyads of the appropriate form. The calculation of the matrix of second partial derivatives of $f_{1}(x, \varepsilon)$ with respect to $x$ and $\varepsilon$ is slightly more complicated by the fact that $T_{i}$ is a composite function of $f(x, \varepsilon)$ and $\varepsilon$. This requires the chain rule to obtain the second derivative matrix. Hence,

$$
D_{\varepsilon x} T_{i}=D_{\varepsilon}\left[\dot{T}_{i} \nabla f_{j}\right]=\dot{T}_{i} \nabla_{\varepsilon x}^{2} f_{j}+\nabla f_{j} \ddot{T}_{1} \nabla_{\varepsilon} f_{j}^{T}+\nabla f_{j} \nabla_{\varepsilon} \dot{T}_{i}
$$

With this, the formulae for the matrix of second partials of $f_{1}$ with respect to $x$ and $\varepsilon$ can be written as in Table 7. The first appearance of these formulae was in deSilva and McCormick (1978).

It should be apparent from $T$ able 7 that an inductive argument paralleling that used in the proof of Theorem 2 would yfeld the fact that $\nabla_{\varepsilon x}^{2}$ can also be written as the sum of dyads. The difference is that for $\nabla_{\varepsilon x}^{2}$, the first vector in the dyads is a gradient with respect to $x$ of a factored-sequence function, and the second vector is a gradient with respect to $\varepsilon$ of a factoredsequence function or a derivative of a single-variable transformation.

This result implies that the submatrices of second-order derivatives which appear in the arrays $\nabla_{y} M, \nabla_{\varepsilon} M, \nabla_{y} N$, and $\nabla_{\varepsilon} N$ on the rhs of $(3.6)$ and in Figures 3, 5, 7, and 9, are all dyadics. What remains is to show that the subarrays of third-order derivatives, in these same four arrays, are triadics. Those subarrays, arising from the block $\nabla^{2} L$ in $M$ and the block $\nabla_{\varepsilon x}^{2}$ in $N$, are $\nabla^{3} L, \nabla_{x \varepsilon x}^{3} L, \nabla_{\varepsilon x x}^{3} L$, and $\nabla_{\varepsilon \varepsilon x}^{3} L$. The first case, $\nabla^{3} L$, is uncomplicated by 
TABLE 5

GRADIENTS OF FACTORABLE FUNCTION FORMS

IN SENSITIVITY ANALYSIS

\begin{tabular}{|c|c|c|}
\hline Rule & $f_{1}$ & $\nabla f_{1}$ \\
\hline 1 & $x_{1}$ & $e_{1}$ \\
\hline $2 a$ & $f_{j}+f_{k}$ & $\nabla f_{j}+\nabla f_{k}$ \\
\hline $2 b$ & $f_{j} \cdot f_{k}$ & $f_{j} \nabla f_{k}+f_{k} \nabla f_{j}$ \\
\hline $2 c$ & $T_{1}$ & $\dot{T}_{1} \nabla f_{j}$ \\
\hline
\end{tabular}

TABLE 6

HESSIANS OF FACTORABLE FUNCTION FORMS

IN SENSITIVITY ANALYSIS

\begin{tabular}{|c|c|c|}
\hline Rule & $f_{1}$ & $\nabla^{2} f_{1}$ \\
\hline 1 & $x_{1}$ & $0_{n \times n}$ \\
\hline $2 a$ & $f_{j}+f_{k}$ & $\nabla^{2} f_{j}+\nabla^{2} f_{k}$ \\
\hline $2 b$ & $f_{j} \cdot f_{k}$ & $f_{j} \nabla^{2} f_{k}+\nabla f_{k} \nabla f_{j}+f_{k} \nabla^{2} f_{j}+\nabla f_{j} \nabla f_{k}$ \\
\hline $2 c$ & $T_{i}$ & $\dot{T}_{1} \nabla^{2} f_{j}+\nabla f_{j} \dot{T}_{i} \nabla f_{j}$ \\
\hline
\end{tabular}


TABLE 7

HESSIANS WITH RESPECT TO $X$ AND $\varepsilon$ OF FACTORABLE

FUNCTION FORMS IN SENSITIVITY ANALYSIS

\begin{tabular}{|c|c|c|}
\hline Rule & $f_{1}$ & $\nabla_{\varepsilon x_{1}}^{2}$ \\
\hline 1 & $x_{1}$ & $o_{n \times r}$ \\
\hline $2 a$ & $f_{j}+f_{k}$ & $\nabla_{\varepsilon x_{j}}^{2}+\nabla_{\varepsilon x}^{2} f_{k}$ \\
\hline $2 b$ & $f_{j} \cdot f_{k}$ & $f_{j} \nabla_{\varepsilon_{x}}^{2} f_{k}+\nabla f_{k} \nabla_{\varepsilon} f_{j}^{T}+f_{k} \nabla_{\varepsilon_{x}}^{2} f_{j}+\nabla f_{j} \nabla_{\varepsilon} f_{k}^{T}$ \\
\hline $2 c$ & $T_{f}\left[f_{j}, \varepsilon\right]$ & $\dot{T}_{1} \nabla_{\varepsilon x}^{2} f_{j}+\nabla f_{j} \ddot{I}_{i} \nabla_{\varepsilon} f_{j}^{T}+\nabla f_{j} \nabla_{\varepsilon} \dot{T}_{1}^{T}$ \\
\hline
\end{tabular}

derivatives with respect to $\varepsilon$, and thus is a triadic by Theorem 4. The proofs for the other cases are in the same vein as the proof of Theorem 2 and require that the formulae for the third derfvatives with respect to $x$ and $\varepsilon$ respectively be derived for the forms in Tables 6 and 7 . These are developed next.

\section{$\frac{3.4 .2 \text { The Triad } 1 c \text { Form of } \nabla_{\varepsilon x x^{f}}^{3}}{3}$}

The first step in showing that $\nabla_{\varepsilon x x} f$ is triadic is to apply the operator $D_{\varepsilon}\{\cdot\}$ to each factorable function form in Table 6. This is straightforward for cases 1 and $2 a$. It is also stralghtforward for $2 b$, but since this case represents the first use of the new notation, it is developed below.

$$
\begin{aligned}
D_{\varepsilon}\left\{f_{j} \nabla^{2} f_{k}+\nabla f_{k} \nabla f_{j}^{T}+f_{k} \nabla^{2} f_{j}+\nabla f_{j} \nabla f_{k}^{T}\right\}= & f_{j} \nabla_{\varepsilon x x}^{3} f_{k}+\nabla^{2} f_{k} \nabla_{\varepsilon}\left\{f_{j}\right\}+\nabla f_{k} \nabla \nabla_{\varepsilon}\left\{\nabla f_{j}^{T}\right\} \\
& +\nabla f_{j}^{T} \nabla_{\varepsilon}\left\{\nabla f_{k}\right\}+f_{k} \nabla_{\varepsilon_{x x}}^{3}+f_{j} f_{j} \nabla_{\varepsilon}\left\{f_{k}\right\} \\
& +\operatorname{sf}_{j} \nabla_{\varepsilon}\left\{\nabla f_{k}\right\}+\nabla f_{k}^{T} \nabla_{\varepsilon}\left\{\nabla f_{j}\right\} .
\end{aligned}
$$


The more complicated case is $2 c$, which is a result of the fact that, as noted earlier, each $T_{\perp}$ (and hence $\dot{T}_{i}$ and $\dot{T}_{i}$ ) is a composite function of $f(x, \varepsilon$ ) and $\varepsilon$, requiring the chain rule to calculate the total derivative. This is shown below.

$$
\begin{aligned}
D_{\varepsilon}\left\{\dot{T}_{1} \nabla^{2} f_{j}+\nabla f_{j} \ddot{T}_{i} \nabla f_{j}\right\}= & \dot{T}_{i} \nabla_{\varepsilon x x}^{3} f_{j}+\nabla^{2} f_{j} D_{\varepsilon}\left\{\dot{T}_{i}\right\}+\nabla \varepsilon_{\varepsilon}\left\{\nabla f_{j}\right\} \ddot{T}_{i} \nabla f_{j} \\
& +\nabla f_{j} D_{\varepsilon}\left\{\ddot{T}_{i}\right\} \nabla f_{j}+\nabla f_{j} \ddot{T}_{i} \nabla_{\varepsilon}\left\{\nabla f_{j}\right\} .
\end{aligned}
$$

However, using the chain rule,

$$
D_{\varepsilon}\left\{\dot{T}_{1}\right\}=\dot{T}_{i} \nabla_{\varepsilon}\left\{f_{j}\right\}+\nabla_{\varepsilon}\left\{\dot{T}_{1}\right\}
$$

and

$$
D_{\varepsilon}\left\{\ddot{T}_{i}\right\}=\ddot{T}_{1} \nabla_{\varepsilon}\left\{f_{j}\right\}+\nabla_{\varepsilon}\left\{\ddot{T}_{i}\right\}
$$

After using ( 3.9$)$, the ths of $(3.8)$ becomes:

$$
\begin{aligned}
& \dot{\mathrm{T}}_{i} \nabla_{\varepsilon \times x^{f}}^{3}+\ddot{T}_{i} \nabla^{2} f_{j} \nabla_{\varepsilon}\left\{f_{j}\right\}+\nabla^{2} f_{j} \nabla_{\varepsilon}\left\{\dot{T}_{i}\right\} \\
& +\nabla_{\varepsilon}\left\{\nabla f_{j}\right\} \ddot{T}_{i} \nabla f_{j}^{T}+\nabla f_{j} \ddot{T}_{i} \nabla_{\varepsilon}\left\{f_{j}\right\} \nabla f_{j}^{T} \\
& +\nabla f_{j} \nabla_{\varepsilon}\left\{\ddot{T}_{i}\right\} \nabla f_{j}^{T}+\nabla f_{j} \ddot{T}_{i} \nabla_{\varepsilon}\left\{\nabla f_{j}^{T}\right\} .
\end{aligned}
$$

These third-derivative formulae for $\nabla_{\text {Exx }}^{3}$ are collected in Table 8 . The proof that $\nabla_{\varepsilon \times x}^{3}$ is triadic is a straightforward application of induction to the factored sequence and mimics the argument used in the proof of Theorem 2 . The result is that $\nabla_{\varepsilon x x^{3}}^{3}$ can be written as the sum of triads of the form

where

$$
\left(\alpha: a_{1} a_{2} a_{3}\right) \text {, }
$$

$$
\begin{aligned}
& a_{1} \text { is }(n \times 1) \text {, } \\
& a_{2} \text { is }(n \times 1) \text {, } \\
& a_{3} \text { is }(x \times 1) \text {, }
\end{aligned}
$$


TABLE 8

THIRD-ORDER TENSORS WITH RESPECT TO $x, x$, AND $\varepsilon$ OF FACTORABLE FUNCT ION FORMS IN SENSITIVITY ANALYSIS

\begin{tabular}{|c|c|c|}
\hline Rule & $f_{1}$ & $\nabla_{\varepsilon \times x f_{1}}^{3}$ \\
\hline 1 & $x_{1}$ & $0_{n \times n \times r}$ \\
\hline $2 a$ & $f_{j}+f_{k}$ & $\nabla_{\varepsilon x x^{f}}^{3}+\nabla_{\varepsilon x x_{k}}^{3}$ \\
\hline $2 b$ & $f_{1} \cdot f_{j}$ & $\begin{array}{l}f_{j} \nabla_{\varepsilon x x}^{3} f_{k}+\nabla^{2} f_{k} \nabla_{\varepsilon}\left\{f_{j}\right\}+\nabla f_{k} \nabla_{\varepsilon}\left\{\nabla f_{j}\right\} \\
+\nabla f_{j}^{T} \nabla_{\varepsilon}\left\{\nabla f_{k}\right\}+f_{k} \nabla_{\varepsilon x x_{j}}^{3}+\nabla^{2} f_{j} \nabla_{\varepsilon}\left\{f_{k}\right\} \\
\quad+\nabla f_{k} \nabla_{\varepsilon}\left\{\nabla f_{k}^{T}\right\}+\nabla f_{k}^{T} \nabla_{\varepsilon}\left\{\nabla f_{j}\right\}\end{array}$ \\
\hline $2 c$ & $T_{1}\left[f_{j}, \varepsilon\right]$ & 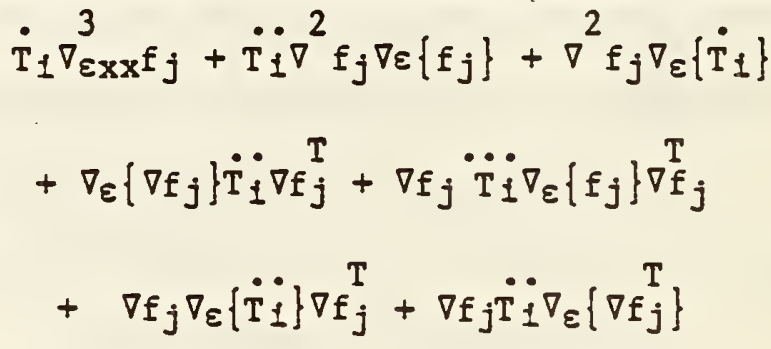 \\
\hline
\end{tabular}

and $\alpha$ is a product of factored sequence functions and f1rst, second, and third derfvatives of the single-variable transformations used in forming the factored sequence. Also, the vector factors $a_{1}$ and $a_{2}$ are gradients with respect to $x$ of a factored sequence function, and a3 is a gradient with respect to $\varepsilon$ of a factored-sequence function or of a first or second derivative with respect to $f$ of one of the single-varlable transformations in the factored sequence. 


\subsubsection{The Triadic Form of $\nabla_{x \varepsilon x^{f}}^{3}$}

Just as in the previous section, the key step in the proof here is to compute the derivatives with respect to $x$ of the forms in Table 7 . Again this process is straightforward, if tedfous, for cases $1,2 a$, and $2 b$, but case $2 c$ presents some complications, and is therefore developed in detail. Thus,

$$
\begin{aligned}
D\left\{\dot{T}_{1} \nabla_{\varepsilon x}^{2} f_{j}+\nabla f_{j} \ddot{T}_{1} \nabla_{\varepsilon} f_{j}+\nabla f_{j} \nabla_{\varepsilon} \dot{T}_{1}\right\} & =\dot{T}_{1} \nabla_{x \varepsilon x}^{3} f_{j}+\nabla_{\varepsilon x} f_{j} D\left\{\dot{T}_{1}\right\}+\nabla\left\{f_{j}\right\} \ddot{T}_{1} \nabla_{\varepsilon} f_{j} \\
& +\nabla f_{j} D\left\{\ddot{T}_{1}\right\} \nabla_{\varepsilon} f_{j}^{T}+\nabla f_{j} \ddot{T}_{i} \nabla\left\{\nabla_{\varepsilon} f_{j} T\right\} \\
& +\nabla f_{j} D\left\{\nabla_{\varepsilon} \ddot{T}_{1}\right\}+\nabla_{\varepsilon} \dot{T}_{i} \nabla\left\{\nabla f_{j}\right\} .
\end{aligned}
$$

But, using the chain rule,

$$
\begin{aligned}
& D\left\{\dot{T}_{i}\right\}=\ddot{T}_{1} \nabla\left\{f_{j}\right\} \\
& D\left\{\ddot{T}_{i}\right\}=\ddot{T}_{1} \nabla\left\{f_{j}\right\}
\end{aligned}
$$

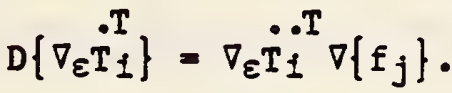

Substituting (3.11) into (3.10) yields the final form given in Table 9, which also contafns the formulae for the other cases. Here too it is easy to see that the same inductive argument used in Theorem 2 results in a triadıc form for $\nabla_{x \varepsilon x^{f}}^{3}$ made of triads of the form

where

$$
\left(\alpha: a_{1} a_{2} a_{3}\right) \text {, }
$$

$$
\begin{aligned}
& a_{1} \text { is }(n \times 1) \text {, } \\
& a_{2} \text { is }(r \times 1) \text {, } \\
& a_{3} \text { is }(n \times 1) \text {, }
\end{aligned}
$$

and $\alpha$ is a product of factored-sequence functions and first, second, and third dertvatives of the single-variable transformations. In this case, $a_{1}$ and $a_{3}$ 
TABLE 9

THIRD-ORDER TENSORS WITH RESPECT TO $x, \varepsilon$, AND $x$ OF FACTORABLE FUNCTION FORMS IN SENSITIVITY ANALYSIS

\begin{tabular}{|c|c|c|}
\hline Rule & $f_{1}$ & $\nabla_{x \in x^{f} \pm}^{3}$ \\
\hline 1 & $x_{1}$ & $0_{n \times r \times n}$ \\
\hline $2 a$ & $f_{j}+f_{k}$ & $\nabla_{x \varepsilon x_{j}}^{3}+\nabla_{x \varepsilon x_{k}}^{3}$ \\
\hline $2 b$ & $f_{j} \cdot f_{k}$ & $\begin{array}{l}f_{j} \nabla_{x \varepsilon_{x} f_{k}}^{3}+\nabla_{\varepsilon_{x} f_{k} \nabla\left\{f_{j}\right\}}^{2}+\nabla f_{k} \nabla\left\{\nabla_{\varepsilon} f_{j}^{T}\right\} \\
+\nabla_{\varepsilon} f_{j}^{T} \nabla\left\{\nabla f_{k}\right\}+f_{k} \nabla_{x \varepsilon_{x} f_{j}}^{3}+\nabla_{\varepsilon_{x} f_{j}}^{2} \nabla\left\{f_{k}\right\} \\
\quad+\nabla f_{j} \nabla\left\{\nabla_{\varepsilon} f_{k}^{T}\right\}+\nabla_{\varepsilon} f_{k}^{T} \nabla\left\{\nabla f_{j}\right\}\end{array}$ \\
\hline $2 c$ & $T_{i}\left[f_{j}, \varepsilon\right]$ & 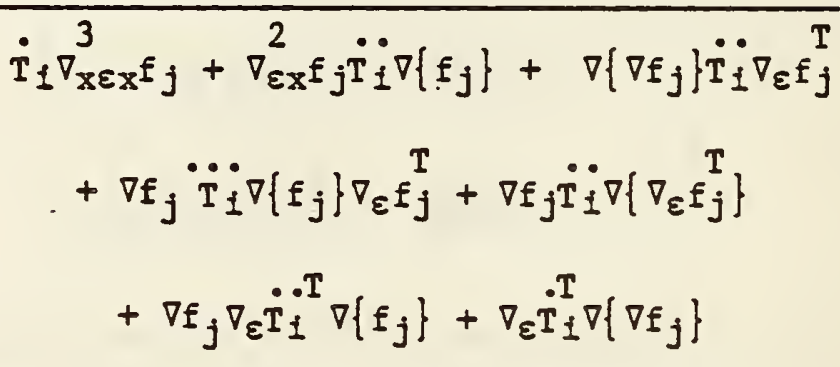 \\
\hline
\end{tabular}

are gradients with respect to $x$ of factored-sequence functions and $a_{2}$ is the gradient with respect to $\varepsilon$ of a factored-sequence function or of a first or second derfvative of a $T_{1}$.

It is Instructive to note that the result in this section could also be obtained more directly by utilizing the triadic structure exh 3

3.4.2 for $\nabla_{\varepsilon x x f}$. Because of the symmetric nature of partial derivatives, that array and the one of this section, $\nabla_{x \varepsilon_{x}}^{3}$, contain the same entries, in different arrangements. In fact, $\nabla_{x \varepsilon x}^{3}$ can be obtained from the former by interchanging the first two vector factors of each triad in its triadic expansion, thereby exhibiting the triadic form for $\nabla_{x \in x}^{3}$ immediately. 


\subsubsection{The Triadic Form of $\nabla_{\varepsilon \varepsilon x}^{3}$ f}

This is the last case to be considered, and also the most difficult of them. As in the previous sections, the formulae for forms $1,2 \mathrm{a}$, and $2 \mathrm{~b}$ are straightforward, but for $2 c$,

$$
\begin{aligned}
& D_{\varepsilon}\left\{\dot{T}_{1} \nabla_{\varepsilon x}^{2} f_{j}+\nabla f_{j} \ddot{T}_{i} \nabla_{\varepsilon} f_{j}^{T}+\nabla f_{j} \nabla_{\varepsilon} \dot{T}_{1}^{T}\right\}=\dot{T}_{1} \nabla_{\varepsilon \varepsilon x}^{3} f_{j}+\nabla_{\varepsilon x}^{2} f_{j} D_{\varepsilon}\left\{\dot{T}_{1}\right\}+\nabla_{\varepsilon}\left\{\nabla_{j}\right\} \ddot{T}_{i} \nabla_{\varepsilon} f_{j}^{T} \\
& +\nabla f_{j} D_{\varepsilon}\left\{\ddot{T}_{i}\right\} \nabla_{\varepsilon} f_{j}^{T}+\nabla f_{j} \ddot{T}_{i} \nabla_{\varepsilon}\left\{\nabla_{\varepsilon} f_{j}^{T}\right\} \\
& +\nabla_{j} D_{\varepsilon}\left\{\nabla_{\varepsilon} \stackrel{T}{T}_{i}\right\}+\nabla_{\varepsilon} \hat{T}_{i} \nabla_{\varepsilon}\left\{\nabla_{j}\right\}
\end{aligned}
$$

Both $D_{\varepsilon}\left\{\dot{T}_{1}\right\}$ and $D_{\varepsilon}\left\{\ddot{T}_{1}\right\}$ were calculated in (3.9). The third spectal computation needed is, using the chain rule again,

$$
D_{\varepsilon}\left\{\nabla_{\varepsilon} \dot{T}_{i}\right\}=\nabla_{\varepsilon} \dot{T}_{i} \nabla_{\varepsilon}\left\{f_{j}\right\}+\nabla_{\varepsilon}\left\{\nabla_{\varepsilon} \dot{T}_{i}\right\}
$$

Substitution gives the formulae for the third partials in Table 10 .

The inductive argument demonstrating the triadic nature of $\nabla_{\varepsilon \varepsilon x}^{3}$ can now go through as before, bearing in mind the assumption in (3.7) that the derivatives with respect to $f$ of the single-variable transformations, $T_{1}$, used In forming the factored sequence are themselves factorable functions in $\varepsilon$. This is required since

$$
\nabla_{\varepsilon}\left\{\nabla_{\varepsilon} \stackrel{\cdot T}{T}_{1}\right\}
$$

appears in the last term of Table 10. Except for orientation In three-space, this is just the Hessian with respect to $\varepsilon$ of the function $\dot{T}_{i}\left[f_{j}(x, \varepsilon), \varepsilon\right]$. So long as $T_{1}$ is factorable in $\varepsilon$, its Hessian is a dyadic in gradients with respect to $\varepsilon$ of its factored sequence, and the statements regarding the triadic nature of $\nabla_{\varepsilon \varepsilon x}^{3}$ go through as before. Thus it is true that $\nabla_{\varepsilon \varepsilon x}^{3}$ can 
TABLE 10

THIRD-ORDER TENSORS WITH RESPECT TO $x, \varepsilon$, AND $\varepsilon$ OF FACTORABLE FUNCTION FORMS IN SENSITIVITY ANALYSIS

\begin{tabular}{|c|c|c|}
\hline Rule & $f_{1}$ & $\nabla_{\varepsilon \varepsilon x}^{3} f_{1}$ \\
\hline 1 & $x_{1}$ & $0_{\text {nXrXr }}$ \\
\hline $2 a$ & $f_{j}+f_{k}$ & $\nabla_{\varepsilon \varepsilon x^{f} j}^{3}+\nabla_{\varepsilon \varepsilon_{x} f_{k}}^{3}$ \\
\hline $2 b$ & $f_{j} \cdot f_{k}$ & $\begin{array}{c}f_{j} \nabla_{\varepsilon \varepsilon_{x}}^{3} f_{k}+\nabla_{\varepsilon x}^{2} f_{k} \nabla_{\varepsilon}\left\{f_{j}\right\}+\nabla_{x} f_{k} \nabla_{\varepsilon}\left\{\nabla_{\varepsilon} f_{j}\right\} \\
+\nabla_{\varepsilon} f_{j}^{T} \nabla_{\varepsilon}\left\{\nabla_{x} f_{k}\right\}+f_{k} \nabla_{\varepsilon \varepsilon x_{j}}^{3}+\nabla_{\varepsilon x}^{2} f_{j} \nabla_{\varepsilon}\left\{f_{k}\right\} \\
+\nabla_{j} \nabla_{\varepsilon}\left\{\nabla_{\varepsilon} f_{k}\right\}+\nabla_{\varepsilon}^{T} f_{k} \nabla_{\varepsilon}\left\{\nabla_{j}\right\}\end{array}$ \\
\hline $2 c$ & $T_{1}\left[f_{j}, \varepsilon\right]$ & 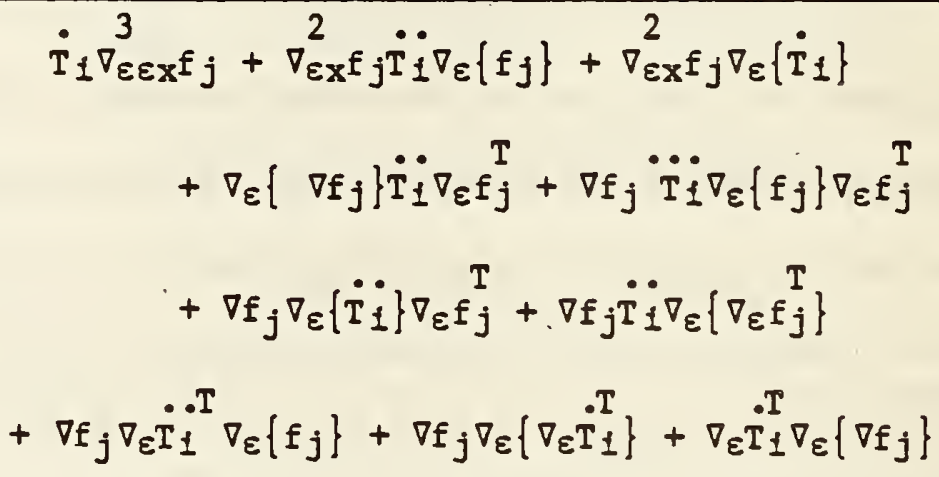 \\
\hline
\end{tabular}

be written as the sum of triads of the form

where

$$
\left(\alpha: a_{1} a_{2} a_{3}\right)
$$

$$
\begin{aligned}
& a_{1} \text { is }(n \times 1) \text {, } \\
& a_{2} \text { is }(r \times 1) \text {, } \\
& a_{3} \text { is. }(r \times 1) \text {, }
\end{aligned}
$$

and $\alpha$ is a product of factors as before, wth additional factors included from the factored sequences for the $\dot{T}_{\perp}$ 's. The last item of note is that here al is 
a gradient with respect to $x$ of a factored-sequence function for $f(x)$, and $a_{2}$ and $a 3$ are gradients with respect to $\varepsilon$ of a factored-sequence function for $f(x)$ or a gradient with respect to $\varepsilon$ of a derivative of a $T_{1}$, or a gradient with respect to $\varepsilon$ of a factored-sequence function or transformation for one of the single-variable transformations in the factored sequence for $f(x)$.

\subsection{Array Multiplication with Generalized Outer Product Matrices}

In the previous section, the polyadic nature of the arrays in (3.6) was exhibited. This section takes up the notion of multiplying these generalized outer product matrices by $Y=\nabla_{\varepsilon} y$, which appears. In (3.6) with two different three-space orientations; viz., $\nabla_{\varepsilon} y$ which is $(p \times r \times 1)$ and $\nabla_{\varepsilon}\{y\}$ which is ( $p \times 1 \times r)$. Multiplication is one area wherefn Factorable Programming, through the natural polyadic nature of the function derivatives involved, offers a potentially great computational saving over alternative approaches. Consider for instance the case of multiplication of a dyad and a matrix,

$$
(a: a b) * F=\left(a a b^{T}\right) F
$$

where $a$ is $(n \times 1), b$ is $(m \times 1), F$ is $(m \times n)$ and $\alpha$ is a scalar. of course one method of computing this is to form (aa) $b^{T}$ which requires $n(m+1)$ multiplications, then to multiply the result by $F$, requiring nm multiplications and $n(m-1)$ additions. A far more efficient way, however, is to form $c=b^{T}$, requiring the same $n m$ multiplications and $n(m-1)$ additions, but now store the result in the dyadic form as

$$
(\alpha: a c)=a \alpha c^{T} \text {, }
$$

thus saving the $n(m+1)$ multiplications required to form the dyad explicitly. In fact, one of the basic tenets of Factorable Programming is that certain 
matrices need never be formed explfcitly, since all required calculations can be performed with the dyadic structures. This of course offers a potentially great computational saving.

It only needs demonstrating, therefore, how to perform the multiplications in (3.6). Consider then multiplication between a generalfzed outer product matrfx of order 3 , a trfad, and a two-dimensional matrix in three-space. Since there are three possfble orfentations for a twodimensfonal matrix in three-space, one could guess that there are six ways to perform the multiplication depending on whether the matrix is pre- or postmultiplying the three dimenstonal array. This is of course the case, and these mitiplications are fllustrated in Figure 10, where in each case the matrix post-multiplies each sfmilarly orfented matrix-slice of the threedimensfonal array. A stmilar sftuation obtains for pre-multiplication.
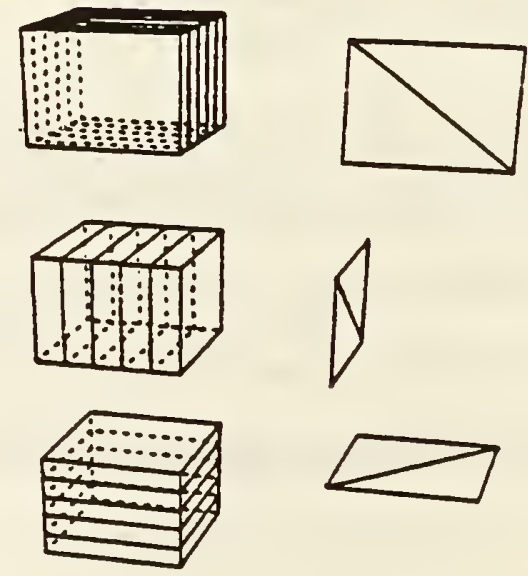

FIGURE 10

THREE TAYS TO MULTIPLY A THREE-DIMENSIONAL ARRAY BY A MATRIX 
Just as with the multiplication of a dyad and a matrix, these three multiplications are simplified when the three-dimensional array is stored as a triadic and it is desfred to store the result as a triadic also. Let one of the triads be ( $\alpha: a b c)$ and the matrix be $F$, and consider the postmultiplication of each each matrix-slice by $F$. Then the result of the multiplications in Figure 10 is just

$$
\begin{aligned}
& \text { 1.) }\left(\alpha: a\left[b^{T} F\right]^{T} c\right), \\
& \text { 11.) }\left(\alpha: a b\left[c^{T} F\right]^{T}\right) \text {, and } \\
& \text { 11.) }\left(\alpha: a b\left[c^{T} F\right]^{T}\right) .
\end{aligned}
$$

(That which appears at first to be an error in (111) is in fact a result of the simple fact that the vector in the third position, associated with the third dimension, is involved in defining two sets of slabs: one set with the vector a in the first dimension, and one set with the vector b in the second dimension).

One use of this ablifty to multiply polyads and matrices is in further explotting the polyadic structure of the matrices in (3.6). It has been shown (McCormick (1983)) that both $M$ and $M^{-1}$ 1n (3.6) can be written in dyadic form. Let, therefore, $-M^{-1}$ in $(3.6)$ be witten in symmetric dyadic form as

$$
-M^{-1}=\sum_{1=1}^{m} a_{1} a_{1} a_{1}=\sum_{1=1}^{m}\left(a_{1}: a_{1} a_{1}\right) .
$$

In the unconstratned case, (3.6) becomes

$$
\nabla_{\varepsilon \varepsilon x}^{2}=-M^{-1} \nabla^{3} L \nabla_{\varepsilon}\{x\} \nabla_{\varepsilon x},
$$

3

and since $1 t$ has been shown that $\nabla \mathrm{L}$ is triadic, let

$$
\nabla^{3} L=\sum_{j=1}^{n}\left(B_{j}: b_{j} b_{j} b_{j}\right) .
$$


Then, for this unconstrained case,

$$
\nabla_{\varepsilon \varepsilon^{x}}^{2}=\sum_{i=1}^{m}\left(\alpha_{1}: a_{1} a_{1}\right) \sum_{j=1}^{m}\left(\beta_{j}: b_{j} b_{j} b_{j}\right) \nabla_{\varepsilon}\{x\} \nabla_{\varepsilon x} .
$$

Application of (11) yields

$$
\nabla_{\varepsilon \varepsilon x}^{2}=\sum_{1=1}^{m}\left(a_{1}: a_{1} a_{1}\right) \sum_{j=1}^{m}\left(\beta_{j}: b_{j}\left[b_{j}^{T} \nabla_{\varepsilon} x\right] b_{j}\right) \nabla_{\varepsilon}\{x\},
$$

and application of (111) yields

$$
\nabla_{\varepsilon \varepsilon^{x}}^{2}=\sum_{1=1}^{m}\left(\alpha_{1}: a_{1} a_{1}\right) \sum_{j=1}^{m}\left(\beta_{j}: b_{j}\left[b_{j}^{T} \nabla_{\varepsilon x}\right]\left[b_{j}^{T} \nabla_{\varepsilon x}\right]\right) .
$$

Similar rules obtain for premultiplication and yield, using the associative law also,

$$
\nabla_{\varepsilon \varepsilon^{x}}^{2}=\sum_{i=1}^{m} \sum_{j=1}^{m}\left(\alpha_{1} \beta_{j}\left[a_{i} b_{j}\right]: a_{1}\left[b_{j}^{T} \nabla_{\varepsilon x}\right]\left[b_{j}^{T} \nabla_{\varepsilon x}\right]\right),
$$

the efficiency of which should be apparent. As an aside, if it were desired, as it was in a recent application of this technique to a problem for the Department of Energy, to produce each frontal slab of this in turn, the $k^{t h}$ of these is given by

$$
\sum_{i=1}^{m} \sum_{j=1}^{n}\left(a_{i} \beta_{j}\left[a_{1}^{T} b_{j}\right] a_{1, k}:\left[b_{j}^{T} \nabla_{\varepsilon} x\right]\left[b_{j}^{T} \nabla_{\varepsilon} x\right]\right),
$$

where, as before, $a_{1, k}$ denotes the $k^{t h}$ element of the vector $a_{1}$. 


\subsection{Parameter Tensors of the Optimal Value Function}

In this section, formulae are developed for the tensors (through order 3) of the optimal value function $f *(\varepsilon)=f[x(\varepsilon), \varepsilon]$ of problem $P(\varepsilon)$ given in (3.1). Armacost and F1acco (1975) were the first to extend basic first-order results for the right-hand-side perturbation problem to the general parametric problem in ( 3.1$)$, and also developed the second-order results given below in Theorem 8. Flacco (1983) gives a complete treatment of all cases for all the variations of (3.1). Our interest is in providing results for the third-order tensor of $f *(\varepsilon)$ and we begin with the first and second-order cases. Theorem 8 Is due to Armacost and Fiacco (1975).

Theorem 8. (First- and Second-Order Changes in $f *(\varepsilon)$ for $P(\varepsilon)_{\bullet}$ )

Let $x$ be a feastble point for $P(\hat{\varepsilon})$ and assume conditions ( 1 ) through (1v) In Theorem 6 . Then, for $\varepsilon$ in a sufficiently small neighborhood of $\hat{\varepsilon}$

a) $f *(\varepsilon)=L^{*}$,

b) $\nabla_{\varepsilon}{ }^{f} *(\varepsilon)=\nabla_{\varepsilon} L$

$$
\begin{aligned}
& =\nabla_{\varepsilon f}-\sum_{i=1}^{m} u_{I} \nabla_{\varepsilon g_{I}} \\
& =\nabla_{\varepsilon f}-u^{T} \nabla_{\varepsilon g} \text {, and }
\end{aligned}
$$

$$
\text { c) } \begin{aligned}
\nabla_{\varepsilon \varepsilon f *}^{2}(\varepsilon) & =\nabla_{y \varepsilon L}^{2} \nabla_{\varepsilon y}+\nabla_{\varepsilon \varepsilon L}^{2} \\
& =\nabla_{x \varepsilon L \nabla_{\varepsilon} x}^{2}-\nabla_{\varepsilon g}{ }^{T} \nabla_{\varepsilon U}+\nabla_{\varepsilon \varepsilon^{L}}^{2}
\end{aligned}
$$

Proof. See Flacco (1983). 
It is easy to see from their forms and from the results given in the previous section that $\nabla_{\varepsilon} f *(\varepsilon)$ is monadic and $\nabla_{\varepsilon \varepsilon}^{2} f *(\varepsilon)$ is dyadic. The result for the third-order tensor of $f *(\varepsilon)$ is given in the following.

\section{Theorem 9. (Third-Order Changes in $f *(\varepsilon)$ for $P(\varepsilon)$. )}

If the conditions of Theorem 8 hold and all third-order partial derivatives In $x$ and third-order partial derivatives in $x$ and $\varepsilon$ exist and are contimuous in $x$ and $\varepsilon$ in a neighborhood of $(\hat{x}, \hat{\varepsilon})$, then

$$
\begin{aligned}
& \nabla_{\varepsilon \varepsilon \varepsilon}^{3} f *(\varepsilon)=\nabla_{y \varepsilon}^{2} L \nabla_{\varepsilon \varepsilon Y}^{2}+\nabla_{y y \varepsilon}^{3} L \nabla_{\varepsilon}\{y\} \nabla_{\varepsilon y}+\nabla_{\varepsilon y \varepsilon}^{3} L \nabla_{\varepsilon} y+\nabla_{\varepsilon \varepsilon \varepsilon}^{3} L+\nabla_{y \varepsilon \varepsilon} L \nabla_{\varepsilon} y \\
& =\nabla_{x \varepsilon L}^{2} \nabla_{\varepsilon \varepsilon x}^{2}+\nabla_{x x \varepsilon}^{3} L \nabla_{\varepsilon}\{x\} \nabla_{\varepsilon x}+\nabla_{u x \varepsilon}^{3} \nabla_{\varepsilon}\{u\} \nabla_{\varepsilon x}+\nabla_{\varepsilon x \varepsilon L}^{3} \nabla_{\varepsilon x} \\
& \left.-\nabla_{\varepsilon g}{ }^{\mathrm{T}} \nabla_{\varepsilon \varepsilon \mathrm{u}}^{2}-\nabla_{\{} \nabla_{\varepsilon \mathrm{g}}^{\mathrm{T}}\right\} \nabla_{\varepsilon}\{\mathrm{x}\} \nabla_{\varepsilon \mathrm{u}}-\nabla_{\varepsilon}\left\{\nabla_{\varepsilon \mathrm{g}}{ }^{\mathrm{T}}\right\} \nabla_{\varepsilon \mathrm{u}}+\nabla_{\varepsilon \varepsilon \varepsilon}^{3} \mathrm{~L},
\end{aligned}
$$

and $\nabla_{\varepsilon \varepsilon \varepsilon}^{3} f *(\varepsilon)$ is a triadic.

$\frac{\text { Proof. Straightforward differentiation of }}{3}$ (c) in Theorem 8 gives the formula for $\nabla_{\varepsilon \varepsilon \varepsilon} f *(\varepsilon)$. The proof of 1ts triadic structure 1s also strafghtforward using the results of the previous sections.

\subsection{Second-Order Sens1tivity Results in Use}

The direct use of first- and second-order sensitivity results is in estimating the solution and multiplier vectors for $P(\varepsilon)$ as the problem is perturbed away from $P(\hat{\varepsilon})$. This estimation is done using the Taylor series approxfmations to two and three terms:

$$
\begin{aligned}
& y(\varepsilon) \sim y(\hat{\varepsilon})+\nabla_{\varepsilon} y(\hat{\varepsilon})(\varepsilon-\hat{\varepsilon}), \\
& y(\varepsilon)=y(\hat{\varepsilon})+\nabla_{\varepsilon} y(\hat{\varepsilon})(\varepsilon-\hat{\varepsilon})+\frac{1}{2} \nabla_{\varepsilon \varepsilon}^{2} y(\hat{\varepsilon})(\varepsilon-\hat{\varepsilon})(\varepsilon-\hat{\varepsilon}),
\end{aligned}
$$


where the multiplication in the third term on the rhs of (3.13) Is understood to be inner product multiplication that reduces the dimension of $\nabla_{\varepsilon \varepsilon y}$.

To 1llustrate this 1 dea as well as some of the other ideas in this section, consider the following parameterfzed nonlinear programming problem:

$$
\underset{x \in R^{n}}{\operatorname{minimize}} f(x, \varepsilon)=x^{T} x+\varepsilon_{1} \exp \left(\varepsilon_{2} a^{T} x\right)
$$

At $\hat{\varepsilon}=0$, the solution by inspection is at $\hat{x}=0$. Since $L(x, u, \varepsilon)=f(x, \varepsilon)$ for this problem, the first-order sensitivity equation, $Y=-M^{-1} N$, reduces to

Also

$$
\nabla_{\varepsilon \times}(\varepsilon)=-\left(\nabla^{2} f\right)^{-1} \nabla_{\varepsilon \times}^{2} f
$$

$$
\begin{gathered}
\nabla f=2 \mathrm{x}+\left[\varepsilon_{1} \varepsilon_{2} \exp \left(\varepsilon_{2} \mathrm{a}^{\mathrm{T}} \mathrm{x}\right)\right] \mathrm{a}, \\
\nabla^{2} \mathrm{f}=2 \mathrm{I}+\mathrm{a}\left[\varepsilon_{1} \varepsilon_{2}^{2} \exp \left(\varepsilon_{2} \mathrm{a}^{\mathrm{T}} \mathrm{x}\right)\right] \mathrm{a}^{\mathrm{T}},
\end{gathered}
$$

and

$$
\nabla_{\varepsilon x^{f}}^{2}=a\left[\exp \left(\varepsilon_{2} a^{T} x\right)\right]\left[\varepsilon_{2}, \varepsilon_{1} \varepsilon_{2} a^{T} x+\varepsilon_{1}\right]
$$

Since $(2 I)^{-1}=\frac{1}{2} I,\left(\nabla^{2} F\right)^{-1}$ can read1ly be obtained using the ShermanWoodbury-Morrison formula (see McCormick (1983), p. 70) that gives the Inverse of a matrix perturbed by a dyad. This formula is

$$
\left(A+u c v^{T}\right)^{-1}=A^{-1}-A^{-1} u\left[c\left(1+v^{T} A^{-1} u c\right)^{-1}\right] v^{T} A^{-1} .
$$

Using this, and letting $c=\varepsilon_{1} \varepsilon_{2} \exp \left(\varepsilon_{2} a^{T} x\right)$,

$$
\left(\nabla^{2} f\right)^{-1}=\left(2 I+a c a^{T}\right)^{-1}=\frac{1}{2} I-a\left[\left(\frac{4}{c}+2 a^{T} a\right)^{-1}\right] a^{T} .
$$


Evaluating these at $\hat{x}=0$ and $\hat{\varepsilon}=0$ yields

and

$$
\left(\nabla^{2} f\right)^{-1}=\frac{1}{2} I
$$

$$
\nabla_{\varepsilon x^{f}}^{2}=0
$$

Therefore

$$
\nabla_{\varepsilon} x(\varepsilon)=0
$$

and the first-order sensitivity analysis approximation (3.12) gives no additional information as the problem is perturbed away from $\hat{x}=0$. This problem is ideally sulted then for a second-order sensitivity analysis using equations (3.13) and (3.6). But since $\nabla_{\varepsilon} x(\varepsilon)=0,(3.6)$ reduces to

$$
\nabla_{\varepsilon \varepsilon^{x}}^{2}(\varepsilon)=-\left(\nabla^{2} f\right)^{-1} \nabla_{\varepsilon \varepsilon \times}^{3} f
$$

Using the formulae developed in the proof of Theorem 3,

$$
\begin{aligned}
\nabla_{\varepsilon \varepsilon x^{f}}^{3}= & \nabla_{\varepsilon}\left(\exp \left[\varepsilon_{2} a^{T} x\right]: a\left[0, \varepsilon_{1} \varepsilon_{2} a^{T} x\right]^{T}\right)+\nabla_{\varepsilon}\left(\exp \left[\varepsilon_{2} a^{T} x\right]: a\left[\varepsilon_{2}, \varepsilon_{1}\right]^{T}\right) \\
= & \left(\exp \left[\varepsilon_{2} a^{T} x\right]: a\left[\varepsilon_{2}, \varepsilon_{1} \varepsilon_{2} a^{T} x\right]^{T}\left[0, a^{T} x\right]^{T}\right) \\
& +\left(\exp \left[\varepsilon_{2} a^{T} x\right]: a\left[0, a^{T} x\right]^{T}\left[\varepsilon_{2}, \varepsilon_{1}\right]^{T}\right) \\
& +\left(\exp \left[\varepsilon_{2} a^{T} x\right]: a\left[\varepsilon_{2}, \varepsilon_{1} \varepsilon_{2} a^{T} x\right]^{T}\left[0, a^{T} x\right]^{T}\right) \\
& +\left(\exp \left[\varepsilon_{2} a^{T} x\right]: a[0,1]^{T}[1,0]^{T}\right) \\
& +\left(\exp \left[\varepsilon_{2} a^{T} x\right]: a[1,0]^{T}[0,1]^{T}\right) .
\end{aligned}
$$


Notice the triadic nature of this tensor and the frequency of occurrence of certain terms. Evaluating these at $\hat{x}=0$ and $\hat{\varepsilon}=0$ yields

$$
\nabla_{\varepsilon \varepsilon x}^{3} f=\left(1: a_{2} e_{1}\right)+\left(1: a_{1} e_{2}\right)
$$

where $e_{i}$ is the $1^{\text {th }}$ unit vector in $R^{n}$. The second partial derivatives with respect to $\varepsilon$ of the solution vector are given by

$$
\begin{aligned}
\nabla_{\varepsilon \varepsilon_{x}(\varepsilon)}^{2} & =-\left(\nabla^{2} f(\hat{x})\right)^{-1} \nabla_{\varepsilon \varepsilon x}^{3} f(\hat{x}) \\
& =-\frac{1}{2} I\left[\left(1: a_{2} e_{1}\right)+\left(1: a_{1} e_{2}\right)\right] \\
& =\left(-\frac{1}{2}: a_{2} e_{1}\right)+\left(-\frac{1}{2}: a_{1} e_{2}\right) .
\end{aligned}
$$

Substituting this into (3.13) gives the second-order estimation

$$
x(\varepsilon)=x(\hat{\varepsilon})+\nabla_{\varepsilon} x(\hat{\varepsilon})(\varepsilon-\hat{\varepsilon})+\frac{1}{2} \nabla_{\varepsilon}^{2} x(\hat{\varepsilon})(\varepsilon-\hat{\varepsilon})(\varepsilon-\hat{\varepsilon}),
$$

which at $\hat{\varepsilon}=0$ becomes

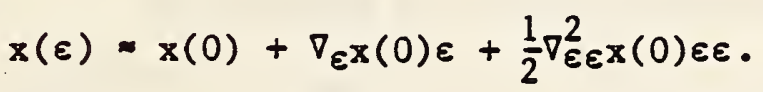

Since $x(0)$ is the solution to the original problem, $x(0)=\hat{x}=0$. Furthermore, $\nabla_{\varepsilon} x(0)$ was shown above to vanish also. Thus

$$
\begin{aligned}
x(\varepsilon) & =\frac{1}{2} \nabla_{\varepsilon \varepsilon x}^{2} x(0) \varepsilon \varepsilon \\
& =\frac{1}{2}\left[\left(-\frac{1}{2}: a e_{2} e_{1}\right)+\left(-\frac{1}{2}: a_{1} e_{2}\right)\right] \varepsilon \varepsilon \\
& =\left(-\frac{1}{4} \varepsilon_{2} \varepsilon_{1}: a\right)+\left(-\frac{1}{4} \varepsilon_{1} \varepsilon_{2}: a\right) \\
& =\left(-\frac{1}{2} \varepsilon_{1} \varepsilon_{2}: a\right) .
\end{aligned}
$$

53 
For a more concrete example of this technique, let $a=(1,2)^{\mathrm{T}}$ and perturb the problem by $\varepsilon=(-1.902, .1)^{\mathrm{T}}$. The solution to this new problem is calculated In Jackson and McCormick (1984) using Halley's third-order method of tangent hyperbolas, and is $(.1, .2)^{\mathrm{T}}$. However, an estimate is given by (3.14), without having to solve the new nonlinear programing problem. The approximation is

$$
\begin{aligned}
x(\varepsilon) & =\left(-\frac{1}{2}(-1.902)(.1):[1,2]^{\mathrm{T}}\right) \\
& =(.0951, .1902)^{\mathrm{T}},
\end{aligned}
$$

which of course is much better than the first-order approximation, $x(\varepsilon)=0$. Another use of the second-order sensitivity formulae is in solving implicitly defined optimization problems. Consider for example the optimization problem

$$
\max _{(p, q)} F(p, q)=x *(p, q)+y *(p, q)+p q
$$

where $\left(x^{*}, y^{*}\right)$ is defined implicitly as the solution of

$$
\min _{(x, y)} G(x, y)=(x-y+3 p q)^{2}+\left(x-(p-q)^{2}\right)^{2}
$$

The analytic solution of this problem is easily obtained as

$$
\begin{gathered}
x *(p, q)=(p-q)^{2} \\
y *(p, q)=(p-q)=(p-q)^{2}+3 p q
\end{gathered}
$$

Substituting, the other problem becomes

$$
\max 2 p^{2}+2 q^{2}
$$

with solution $\left(p^{*}, q^{*}\right)=(0,0)$.

Let $\varepsilon=(p, q)^{T}$. The solution of the maximization problem will be accomplished in one iteration of Newton's method using the second-order sensitivity formulas. 
It is required to compute

$$
\varepsilon_{0}-\left[\nabla_{\varepsilon \varepsilon}^{2} F\left(\varepsilon_{0}\right)\right]^{-1} \nabla \varepsilon F\left(\varepsilon_{0}\right)
$$

Now

$$
\nabla_{\varepsilon} F=\nabla_{\varepsilon} x^{*}+\nabla_{y} *+[q, p]^{T}
$$

And

$$
\nabla_{\varepsilon \varepsilon F}^{2}=\nabla_{\varepsilon \varepsilon X^{*}}^{2}+\nabla_{\varepsilon \varepsilon Y^{*}}^{2}+\left|\begin{array}{ll}
0 & 1 \\
1 & 0
\end{array}\right|
$$

Let $z$ denote $[x, y]^{T}$. Then from first-order sensitivity analysis

$$
\nabla_{\varepsilon} z^{*}=-\left(\nabla_{\varepsilon \varepsilon G)}^{2} \nabla_{\varepsilon z G}\right.
$$

Now

and

$$
\nabla_{z_{Z} G}^{2}=\left.\right|_{-1} ^{1}|(2)[1,-1]+| \begin{aligned}
& 1 \\
& 0
\end{aligned} \mid(2)[1,0]
$$

$$
\nabla_{\varepsilon z^{G}}^{2}=\left|\begin{array}{c}
1 \\
-1
\end{array}\right|(2)[3 q, 3 p]+\left|\begin{array}{c}
1 \\
0
\end{array}\right|(4)(p q)[1,-1]
$$

The most natural representation of $\left(\nabla_{\mathrm{zz}_{2} G}^{2}\right)^{-1}$ is in dyadic form and is

$$
\left|\begin{array}{c}
0 \\
-1
\end{array}\right|(1 / 2)[0,-1]+\left|\begin{array}{c}
1 \\
1
\end{array}\right|(1 / 2)[1,1]
$$

The second-order sensitivity formula (3.6) can be rewritten (conceptua11y) In this case as

$$
\nabla_{\varepsilon \varepsilon z^{*}}^{2}=-\left(\nabla_{\varepsilon \varepsilon G}^{2}\right)^{-1}\left[\left(\nabla_{\varepsilon z z^{G}}^{3}\right)\left(\nabla_{\varepsilon z^{*}}\right)+\left(\nabla_{z z z^{G}}^{3}\right)\left(\nabla_{\varepsilon z^{*}}\right)^{2}+\nabla_{\varepsilon \varepsilon z^{G}}^{3}+\left(\nabla_{z \varepsilon z^{G}}^{3}\right)\left(\nabla_{\varepsilon z^{*}}\right)\right]
$$

For this problem the only term multiplying the inverse which does not vanish 3 is $\nabla_{\varepsilon \in z_{Z} G}, a(2 \times 2 \times 2)$ matrix. 
Its triadic form is

$$
\begin{aligned}
& \nabla_{\varepsilon \varepsilon Z^{G}}^{3}=\left(6:[1,-1]^{\mathrm{T}}[1,0]^{\mathrm{T}}[0,1]^{\mathrm{T}}\right)+\left(6:[1,-1]^{\mathrm{T}}[0,1]^{\mathrm{T}}[1,0]^{\mathrm{T}}\right) \\
& +\left(-4:[1,0]^{\mathrm{T}}[1,-1]^{\mathrm{T}}[1,-1]^{\mathrm{T}}\right) \text {. }
\end{aligned}
$$

Suppose $\left[p_{0}, q_{0}\right]=[2,1]$. Then

$$
\begin{gathered}
\nabla_{\varepsilon} z_{0}^{*}=-\left|\begin{array}{c}
0 \\
-1
\end{array}\right|(1 / 2)[0,-1]+\left|\begin{array}{l}
1 \\
1
\end{array}\right|(1 / 2)[1,1]+\left|\begin{array}{r}
1 \\
-1
\end{array}\right|(2)[3,6] \\
+\left|\begin{array}{l}
1 \\
1
\end{array}\right|(4)[1,-1] .
\end{gathered}
$$

Theref ore

and thus

$$
\nabla_{\varepsilon x_{0}}{ }^{*}=[2,-2]^{\mathrm{T}}, \nabla_{\varepsilon y_{0}}{ }^{*}=[5,4]^{\mathrm{T}},
$$

$$
\nabla_{\varepsilon} F_{0}=[2,-2]^{T}+[5,4]^{T}+[1,2]^{T}=[8,4]^{T} \text {. }
$$

The second-order sensitivity formulas yield

$$
\begin{aligned}
-\left[\nabla_{\mathbf{z}}^{2} G\right]^{-1}\left[\nabla_{\left.\varepsilon \varepsilon z^{G}\right]}^{3}=\right. & -\left[\left(1 / 2:[0,-1]^{\mathrm{T}}[0,-1] \mathrm{T}\right)+\left(1 / 2:[1,1]^{\mathrm{T}}[1,1] \mathrm{T}\right)\right] \\
& *\left[\left(6:[1,-1]^{\mathrm{T}}[1,0]^{\mathrm{T}}[0,1]^{\mathrm{T}}\right)+\left(6:[1,-1]^{\mathrm{T}}[0,1]^{\mathrm{T}}[1,0] \mathrm{T}\right)\right. \\
& \left.+\left(-4:[1,0]^{\mathrm{T}}[1,-1]^{\mathrm{T}}[1,-1] \mathrm{T}\right)\right] .
\end{aligned}
$$

To 1llustrate the computation, one of the s1x product terms will be computed:

$$
\begin{aligned}
\left(-1 / 2:[0,1]^{\mathrm{T}}[0,-1]^{\mathrm{T}}\right) *\left(6:[1,-1]^{\mathrm{T}}[1,0]^{\mathrm{T}}[0,1]^{\mathrm{T}}\right) \\
=\left(-(1)(1 / 2)(6)[0,-1][1,-1]^{\mathrm{T}}:[0,-1]^{\mathrm{T}}[1,0]^{\mathrm{T}}[0,1]^{\mathrm{T}}\right) \\
=\left(-3:[0,1]^{\mathrm{T}}[1,0]^{\mathrm{T}}[0,1]^{\mathrm{T}} .\right.
\end{aligned}
$$

In all, the resulting triadic form has the following terms:

$$
\begin{aligned}
& \left(-3:[0,-1]^{\mathrm{T}}[1,0]^{\mathrm{T}}[0,1]^{\mathrm{T}}\right) \\
+ & \left(-3:[0,-1]^{\mathrm{T}}[0,1]^{\mathrm{T}}[1,0]^{\mathrm{T}}\right) \\
+ & \left(2:[1,1]^{\mathrm{T}}[1,-1]^{\mathrm{T}}[1,-1]^{\mathrm{T}}\right)
\end{aligned}
$$


From this,

and

$$
\nabla_{\varepsilon \varepsilon_{0} x_{0}^{*}}^{2}=\left(2:[1,-1]^{T}[1,-1]^{T}\right)
$$

$$
\nabla_{\varepsilon \varepsilon \mathrm{Y}_{0}}^{2}=\left(2:[1,-1]^{\mathrm{T}}[1,-1]^{\mathrm{T}}\right)+\left(3:[0,1]^{\mathrm{T}}[1,0]^{\mathrm{T}}\right)+\left(3:[1,0]^{\mathrm{T}}[0,1]^{\mathrm{T}}\right)
$$

Thus

$$
\nabla_{\varepsilon \varepsilon F_{0}}^{2}=\nabla_{\varepsilon \varepsilon x_{0}}^{2}+\nabla_{\varepsilon \varepsilon y_{0} *}^{2}+\left|\begin{array}{ll}
0 & 1 \\
1 & 0
\end{array}\right|=\left|\begin{array}{ll}
4 & 0 \\
0 & 4
\end{array}\right|
$$

Combining,

$$
\varepsilon_{0}-\left(\nabla_{\varepsilon \varepsilon F_{0}}^{2}\right)^{-1}\left(\nabla_{\varepsilon} F_{0}\right)=\left|\begin{array}{l}
2 \\
1
\end{array}\right|-\left|\begin{array}{ll}
4 & 0 \\
0 & 4
\end{array}\right|^{-1}\left|\begin{array}{l}
8 \\
4
\end{array}\right|=\left|\begin{array}{l}
0 \\
0
\end{array}\right|
$$

as destred. 
R.L. Armacost and A.V. Fłacco, "Computational experfence in sensitivity analysts for nonlinear programming," Mathematical Programming 6, $(1974), 301-326$.

R.L. Armacost and A.V. Flacco, "Sensitfvity analysis for parametric nonlinear programing using penalty methods," In Computers and Mathematical Programming, National Bureau of Standards Special Publication 502, (1978) 261-269.

G.A. Bliss, Lectures on the calculus of variations, (Universtty of Chicago Press, Ch士cago, 1946).

R.S. Dembo, "Sensitivity analysis in geometric programming," JOTA, 37 (1982) $1-21$.

A. DeS 1 va and G. P. McCormick, "Sensttıvity analysis in nonlinear programming using factorable symbolic Input," Technical Report T-365, The George Washington UnIvers1ty, Institute for Management Sclence and Engineering, (Washington, DC, 1978).

G. Emami, "Evaluating strategies for Newton's method using a numerically stable generalized Inverse algor 1 thm," Dissertation, The George Washington Unfvers 1 ty, Department of Operatıons Research, (Washington, DC, 1978).

A.V. F1acco and G.P. McCormick, Nonlinear Programing: Sequentlal Unconstrained Minimzation Techniques, (W1ley, New York, 1968).

A.V. Flacco, Sensitivity analysis for nonlinear programming using penalty methods," Math. Prog., 10 (1976) 287-311.

A.V. Fłacco, "Nonlinear programming sensitivity analysis results us ing strong second order assumptions," In Numerical Optimization of Dynamic Systems (L.C.W. Dixon and G.P. Szego, eds.), (North-Holland, Amsterdam, 1980 $327-348$.

A.V. Fłacco, Introduction to Sensitivity and Stability Analysis in Nonlinear Programming, (Academic Press, New York, 1983).

J.J. Filliben, "DATAPLOT--an interactive high level language for graphics, nonlinear fitting, data analysis and mathematics, "Computer Graphics, 15 (1981) 199-213.

A. Ghaem, and G.P. McCormick, "Factorable symbolic SUMT: What is it? How is it used?", Technical Report T-402, The George Washington University Institute for Management Sclence and Engineering (Washington, DC, 1979).

F. Ghotb, "Newton's method for Hnearly constrained optimization problems," Dissertation, The George Washington University, Department of Operations Research, (Washington, DC, 1980). 
A. Graham, Kronecker Products and Matrix Calculus with Applications, (Wiley, New York, 1981).

K.E. Hillstrom, "JAKEF - a portable symbolic differentiator of functions given by algorithms," Technical report ANL-82-48, Argonne National Laboratory, (Argonne, IL, 1982).

R.H.F. Jackson, "Tensors, Polyads, and High-Order Methods in Factorable Programming," Dissertation, The George Washington University, Department of Operations Research, (Washington, DC, 1983).

R.H.F. Jackson and G.P. McCormfck, "The polyadic structure of factorable function tensors with application to high-order minimization techniques," JOTA, to appear, (1984).

J. Kyparisis, "Sensitivity and stability for nonlinear and geometric programming: theory and applications. Dissertation, The George Washington University, Department of Operations Research (Washington, DC, 1983).

A. Linneman, "Higher-order necessary conditions for infinite and semiinfinite optimization," JOTA, 38 (1982) 483-511.

W.H. Marlow, Mathematics for Operations Research, (Wiley, New York, 1978).

G.P. McCormick, "Second order conditions for constrained minima," SIAM J. App1. Math., 15 (1967) 37-47.

G.P. McCormick, "A mint-manual for use of SUMr computer program and the factorable programing language," Technical report SOL-74-15, Stanford University Department of Operations Research, (Stanford, CA, 1974).

G.P. McCormick; Nonlinear Programming: Theory, Algorithms and Applications, (Wiley, New York, 1983).

A. Miele and S. Gonzalez, "On the comparative evaluation of algorithms for mathematical programming problems," in 0.L. Mangasarian, et al., (eds.): Nonlinear Programming 3, (Academic Press, New York, 1978) $\frac{337-359 .}{}$

W.C. Mylander, R. Holmes, and G.P. McCormick, "A guide to SUMT-Vers1on 4: The computer program implementing the sequential unconstrained minfmization technique for nonlinear programming," Technical Report RAC-P-63, Research Analysis Corporation (McLean, VA, 1971).

L. Pennisi, "An indirect proof of the problem of Lagrange with differential Inequalities as added side conditions," Trans. Am. Math. Soc., 74 (1953), $177-198$.

R.E. Pugh, "A language for nonlinear programming problems" Math. Prog., 2 (1972) 176-206. 
-M.E. Shayan, "A methodology for comparing algorithms and a method for computing $m^{\text {th }}$ order directional derivatives based on factorable programing," Dissertation, The George Washington University, Department of Operations Research, (Washington, DC, 1978).

A. Sofer, "Computationally efficlent techniques for generalized inversion in nonlinear programing," Dissertation, The George Washington University, Department of Operations Research, (Washington, DC, 1983). 
4. TITLE ANO SUBTITLE

Polyadic Third-order Lagrangian Tensor Structure and

Second-Order Sensitivity Analysis with Factorable Functions

5. AUTHOR(S)

Richard H. F. Jackson and Garth P. McCormick

6. PERFORMING ORGANIZATION (If joint or other than NBS, see instructions)

7. ContracdGrant No.

NATIONAL BUREAU OF STANDARDS

DEPARTMENT OF COMMERCE

WASHINGTON, D.C. 20234

9. SPONSORING ORGANIZATION NAME AND COMPLETE ADDRESS (Street, City. Stote, ZIP)

Operations Research Division

Center for Applied Mathematics

National Bureau of Standards

Gaithersburg, MD 20899

10. SUPPLEMENTARY NOTES

Document describes a computer program; SF-185, FIPS Software Summary, is attached.

11. ABSTRACT (A 200-word or less foctual summary of most significant information. If document includes a significant bibliography or literature survey, mention it here)

Second-order sensitivity analysis methods are developed for analyzing the behavior of a local solution to a constrained nonlinear optimization problem when the problem functions are perturbed slightly. Specifically, formulas involving third-order tensors are given to compute second derivatives of components of the local solution with respect to the problem parameters. When in addition, the problem functions are factorable, it is shown that the resulting tensors are polyadic in nature.

12. KEY WORDS (Six to twelve entries: alphabetical order; capitalize only proper names; and separate key words by semicolons Second-order sensitivity analysis; high-order methods; $N^{\text {th }}$ derivatives; polyads; tensors

13. AVAILABILITY

$X$ Unlimited

For Official Distribution. Do Not Release to NTIS

Order From Superintendent of Documents, U.S. Government Printing Office, Washington. D.C. 20402.

14. NO. OF PRINTED PAGES

\section{7}

15. Price

$\$ 10.00$

X] Order From National Technical Information Service (NTIS). Springfield, VA. 22161 


